\title{
Constraining the Higgs portal with antiprotons
}

\author{
Alfredo Urbano ${ }^{a}$ and Wei Xue $\mathrm{e}^{b, c, a}$ \\ ${ }^{a}$ SISSA - International School for Advanced Studies, \\ via Bonomea 265, I-34136, Trieste, Italy \\ ${ }^{b}$ Center for Theoretical Physics, Massachusetts Institute of Technology, \\ Cambridge, MA 02139, U.S.A. \\ ${ }^{c}$ INFN - Sezione di Trieste, \\ via Bonomea 265, I-34136, Trieste, Italy \\ E-mail: alfredo.urbano@sissa.it, weixue@mit.edu
}

ABstRACT: The scalar Higgs portal is a compelling model of dark matter (DM) in which a renormalizable coupling with the Higgs boson provides the connection between the visible world and the dark sector. In this paper we investigate the constraint placed on the parameter space of this model by the antiproton data. Due to the fact that the antiprotonto-proton ratio has relative less systematic uncertainties than the antiproton absolute flux, we propose and explore the possibility to combine all the available $\bar{p} / p$ data. Following this approach, we are able to obtain stronger limits if compared with the existing literature. In particular, we show that most of the parameter space close to the Higgs resonance is ruled out by our analysis. Furthermore, by studying the reach of the future AMS02 antiproton and antideuteron data, we argue that a DM mass of $\mathcal{O}(150) \mathrm{GeV}$ offers a promising discovery potential. The method of combining all the antiproton-to-proton ratio data proposed in this paper is quite general, and can be straightforwardly applied to other models.

Keywords: Higgs Physics, Beyond Standard Model

ARXIV EPRINT: 1412.3798 


\section{Contents}

1 Introduction 1

2 Setup: the scalar Higgs portal $\quad 2$

2.1 Relic density 3

2.2 LHC bound 5

2.3 Direct detection constraint 5

3 Antiprotons: background vs. signal $\quad 6$

3.1 Selection of propagation models $\quad 6$

3.2 Antiproton bound on the scalar Higgs portal model 9

4 Results $\quad 10$

$\begin{array}{lll}4.1 & \text { On the impact of different propagation models } & 10\end{array}$

$\begin{array}{lll}4.2 & \text { On the impact of different DM density profiles } & 14\end{array}$

$\begin{array}{llr}5 & \text { Future perspectives } & 19\end{array}$

$\begin{array}{lll}5.1 \text { AMS-02 } & 19\end{array}$

5.2 Antideuteron 20

6 Conclusions and outlook $\quad 22$

$\begin{array}{ll}\text { A Spin-1/2 Higgs portal } & 23\end{array}$

\section{Introduction}

Since the discovery of the first negative proton in 1955 [1], antiprotons have become a fundamental pillar in experimental high-energy physics, both in collider physics and astrophysics. In particular, antiprotons play a starring role in the context of indirect detection of dark matter (DM): they are copiously produced in the final stages of DM annihilations into Standard Model (SM) particles as a consequence of showering and hadronization processes. This is in particular true considering DM annihilation into hadronic channels, like for instance annihilation into $\bar{b} b$. However, thanks to the electroweak radiative corrections, this is also true for DM annihilations into leptonic channels, providing that the DM mass is around the $\mathrm{TeV}$ scale $[2-4]$.

Furthermore, the astrophysical background plaguing this potential DM signal is relatively well understood. In a standard scenario it mostly consists in secondary antiprotons originated from the interactions of primary cosmic-ray protons, produced in supernova remnants, with the interstellar gas. For these reasons the antiproton channel is considered one of the most promising probes to shed light on the true nature of DM [5-8]. 
However, all the experimental data collected so far show a fairly good agreement with the predictions of the astrophysical background, usually computed by means of dedicated codes such as GALPROP or DRAGON. Overturning the previous perspective, this negative results is often exploited to place strong bounds on the annihilation cross-section of DM into SM particles (see, e.g., refs. [9-19, 21, 22]). Following this line, in this paper we explore the constraining power of the antiproton data considering as a benchmark example the so-called Higgs portal DM model [23-26]. Despite its simplicity, in fact, this model offers a rich phenomenology, and it provides a simple and motivated paradigm of DM. ${ }^{1}$

The aim of this paper is twofold. On the one hand, we extract our bounds focusing the analysis on the experimental data describing the antiproton-to-proton ratio instead of (as customary in the literature) the absolute antiproton flux. In this way we can get rid of the systematic uncertainties that usually preclude the comparison between data taken by different experiments. On the other one, we compare, in the context of the Higgs portal model and in a wide range of DM mass, our results with the bounds obtained considering the invisible Higgs decay width and the spin-independent DM-nucleon cross-section. The purpose of this comparison is to highlight the regions of the parameter space in which the antiproton data give the most stringent limits.

This paper is organized as follows. In section 2, we briefly introduce the scalar Higgs portal model. In section 3, we discuss all the relevant aspects of our analysis; in particular, we present in detail the computation of the $\bar{p} / p$ flux considering both the standard astrophysical background and the DM signal. In section 4 we present our results, and in section 5 we discuss future prospects. Finally, we conclude in section 6 . In appendix A, we generalize our results to the fermionic Higgs portal model.

\section{Setup: the scalar Higgs portal}

The Lagrangian of the scalar Higgs portal model that we consider in this work has the following structure [23-26]

$$
\mathcal{L}_{\mathrm{HP}}=\mathcal{L}_{\mathrm{SM}}+\frac{1}{2}\left(\partial_{\mu} S\right)\left(\partial^{\mu} S\right)-\frac{m_{0}^{2}}{2} S^{2}-\frac{\lambda_{\mathrm{S}}}{2}|H|^{2} S^{2},
$$

where $\mathcal{L}_{\mathrm{SM}}$ is the SM Lagrangian, $\lambda_{\mathrm{S}}$ is the Higgs portal coupling, and the real field $S$ is a scalar gauge singlet with mass - after electroweak symmetry breaking — given by $m_{\mathrm{S}}=\left(m_{0}^{2}+\lambda_{\mathrm{S}} v^{2} / 2\right)^{1 / 2} ; H$ is the SM Higgs doublet with vacuum expectation value (vev) $\langle H\rangle=v / \sqrt{2}=174 \mathrm{GeV}$.

The relevant parameter space of the model is the two-dimensional plane $\left(m_{\mathrm{S}}, \lambda_{\mathrm{S}}\right)$. After electroweak symmetry breaking the Higgs portal coupling generates the trilinear vertex $\mathcal{L}_{\mathrm{hS}^{2}}=\lambda_{\mathrm{S}} v h S^{2} / 2$, where $h$ is the physical Higgs boson; this interaction is responsible for all the phenomenological properties of the model since via this vertex the DM particle communicates with all the SM species. In this paper, we focus on the possibility that the scalar field $S$ plays the role of cold DM in the Universe.

In appendix A, we will analyze a different type of Higgs portal with fermionic DM.

\footnotetext{
${ }^{1}$ See for instance ref. [27] for a recent application.
} 

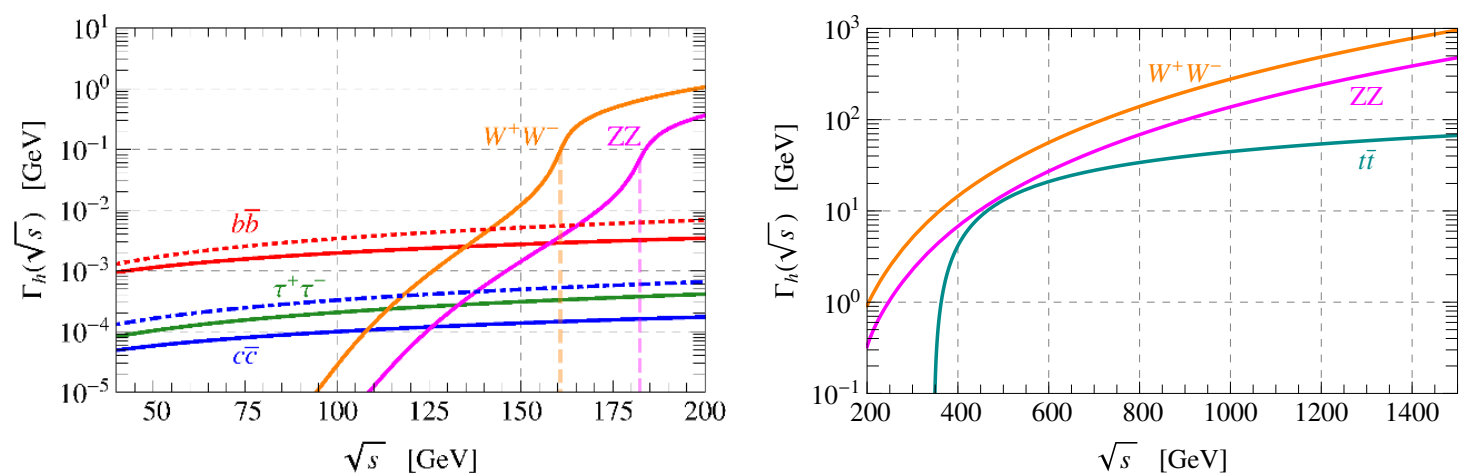

Figure 1. Off-shell decay width of the Higgs boson $\Gamma_{\mathrm{h}}(\sqrt{s})$ in the energy interval $\sqrt{s} \in[40,200] \mathrm{GeV}$ obtained using the public code HDECAY (left panel, solid lines), including the NLO QCD corrections to the Higgs decay into quarks and the Higgs decay modes into off-shell gauge bosons. We show separately the most important contributions relevant for the computation of the annihilation cross-section in eq. (2.2). The impact of the radiative corrections is proven by the comparison with the corresponding tree-level expressions (tree-level Higgs decay width into $b \bar{b}$, dotted line, and $c \bar{c}$, dot-dashed line); we also show the $W^{+} W^{-}$and $Z Z$ kinematical thresholds (vertical dashed lines) to emphasize the importance of the Higgs decay modes into off-shell gauge bosons in the region close to the Higgs resonance, $\sqrt{s}=126 \mathrm{GeV}$. At higher energies, $\sqrt{s}>200 \mathrm{GeV}$, we compute $\Gamma_{\mathrm{h}}(\sqrt{s})$ analytically (right panel).

\subsection{Relic density}

Through the exchange of the Higgs in the s-channel, two DM particles can annihilate into all the SM final states that are kinematically allowed by the value of the DM mass, $m_{\mathrm{S}}$. The annihilation cross-section times the relative velocity $v_{\text {rel }}$ of the two annihilating DM particles takes the remarkably simple form

$$
\sigma v_{\text {rel }}=\frac{2}{\sqrt{s}}\left[\frac{\lambda_{\mathrm{S}}^{2} v^{2}}{\left(s-m_{h}^{2}\right)^{2}+\Gamma_{\mathrm{h}, \mathrm{S}}^{2} m_{h}^{2}}\right] \Gamma_{\mathrm{h}}(\sqrt{s}),
$$

where the square of the total energy in the c.o.m. frame is $s=4 m_{\mathrm{S}}^{2} /\left(1-v_{\text {rel }}^{2} / 4\right)$. In eq. $(2.2) \Gamma_{\mathrm{h}}(\sqrt{s})$ is the off-shell decay width of the Higgs boson (with $m_{h}^{*}=\sqrt{s}$ ), summed over all the SM final states. We use the public code HDACAY [28] to compute the width $\Gamma_{\mathrm{h}}(\sqrt{s})$. In this way we are able to include $\left.i\right)$ the $\mathcal{O}\left(\alpha_{s}\right)$ NLO QCD radiative corrections to the Higgs decay into quarks and $i i)$ the Higgs decay modes into off-shell gauge bosons. The importance of these radiative effects has been emphasized in ref. [29]. We plot in the left panel of figure 1 the function $\Gamma_{\mathrm{h}}(\sqrt{s})$ in the energy interval $\sqrt{s} \in[40,200] \mathrm{GeV}$ (left panel); we separate the most important contributions, namely the Higgs decays into SM quarks $(b \bar{b}$ and $c \bar{c})$ and tau leptons as well as electroweak gauge bosons $\left(W^{+} W^{-}\right.$and $Z Z$ ). The importance of the radiative corrections is evident from the comparison with the corresponding tree level expressions (dashed lines in figure 1).

Going towards higher energies, there is an important issue to keep in mind. In the SM the Higgs quartic coupling $\lambda$ is a function of the Higgs mass, i.e. $\lambda\left(m_{h}\right)=m_{h}^{2} / 2 v^{2}$ and, as a consequence, $\lambda(126) \simeq 0.13$. In the computation of the off-shell decay width 
$\Gamma_{\mathrm{h}}(\sqrt{s})$, some electroweak corrections involving the quartic coupling are overestimated at high energy, growing like $\lambda(\sqrt{s})=s / 2 v^{2}$. In order to get rid of this issue, for $\sqrt{s}>200 \mathrm{GeV}$ we compute $\Gamma_{\mathrm{h}}(\sqrt{s})$ analytically; we show the corresponding values for annihilation into $W^{+} W^{-}, Z Z$ and $t \bar{t}$ in the right panel of figure 1 in the energy interval $\sqrt{s} \in[200,1500] \mathrm{GeV}$. Finally, notice that above the kinematical threshold $\sqrt{s}>2 m_{h}=252 \mathrm{GeV}$ DM annihilation into two Higgses is kinematically allowed; the corresponding annihilation cross-section, however, cannot be recast in the form described by eq. (2.2) since, in addition to the schannel exchange of the Higgs, also t- and u-channel diagrams in which the DM particle is exchanged contribute to the amplitude. We include this channel computing the crosssection analytically (see, e.g., ref. [30]).

In eq. (2.2) $\Gamma_{\mathrm{h}, \mathrm{S}}$ represents the on-shell decay width of the Higgs boson and it consists of two pieces, namely $\Gamma_{\mathrm{h}, \mathrm{S}} \equiv \Gamma_{\mathrm{h}}^{\mathrm{SM}}+\Gamma_{\mathrm{h} \rightarrow \mathrm{SS}} ; \Gamma_{\mathrm{h}}^{\mathrm{SM}}=4.217 \mathrm{MeV}$ is the $\mathrm{SM}$ contribution while $\Gamma_{\mathrm{h} \rightarrow \mathrm{SS}}$ is the decay width describing the process $h \rightarrow S S$, kinematically allowed if $m_{\mathrm{S}}<m_{h} / 2$. The explicit expression of $\Gamma_{\mathrm{h} \rightarrow \mathrm{SS}}$ is discussed in the context of the LHC bound (see section 2.2, eq. (2.8)).

The thermally averaged annihilation cross-section is given by

$$
\left\langle\sigma v_{\mathrm{rel}}\right\rangle=\int_{4 m_{\mathrm{S}}^{2}}^{\infty} d s \frac{s \sqrt{s-4 m_{\mathrm{S}}^{2}} K_{1}(\sqrt{s} / T)}{16 T m_{\mathrm{S}}^{4} K_{2}^{2}\left(m_{\mathrm{S}} / T\right)} \sigma v_{\mathrm{rel}},
$$

where $T$ is the temperature and $K_{\alpha=1,2}$ are the modified Bessel functions of second kind. We numerically solve the Boltzmann equation describing the evolution of the number density $n(x)$ of the DM particles during the expansion of the Universe, being $x \equiv m_{\mathrm{S}} / T$. In terms of the yield $\mathrm{Y}(x)=n(x) / s(x)$, where $s(x)$ is the entropy density, this equation reads

$$
\frac{d \mathrm{Y}}{d x}=-Z(x)\left[\mathrm{Y}^{2}(x)-\mathrm{Y}_{\mathrm{eq}}^{2}(x)\right]
$$

where

$$
Z(x) \equiv \sqrt{\frac{\pi}{45}} \frac{m_{\mathrm{S}} M_{\mathrm{PL}}}{x^{2}} \sqrt{g_{*}(T)}\left\langle\sigma v_{\mathrm{rel}}\right\rangle(x) .
$$

$M_{\mathrm{PL}}=1.22 \times 10^{19} \mathrm{GeV}$ is the Planck mass, and $g_{*}(T)$ is the number of relativistic degrees of freedom. At the equilibrium, we have

$$
\mathrm{Y}_{\mathrm{eq}}(x)=\frac{45}{4 \pi^{4}} \frac{x^{2}}{h_{\mathrm{eff}}(T)} K_{2}(x),
$$

where $h_{\mathrm{eff}}(T)$ is the effective entropy. ${ }^{2}$ The integration of the Boltzmann equation gives the yield today, $\mathrm{Y}_{0}$, which is related to the DM relic density through

$$
\Omega_{\mathrm{DM}} h^{2}=\frac{2.74 \times 10^{8} m_{\mathrm{S}} \mathrm{Y}_{0}}{\mathrm{GeV}} .
$$

The Planck collaboration has recently reported the value [33] $\Omega_{\mathrm{DM}} h^{2}=0.1199 \pm 0.0027$ ( $68 \%$ C.L.); we compute the relic density according to eq. (2.7), and we impose to match the measured value.

\footnotetext{
${ }^{2}$ We include in our numerical analysis the temperature dependence in $g_{*}(T)$ and $h_{\mathrm{eff}}(T)$; we extract the corresponding functions from the DarkSUSY code [31, 32].
} 
Before proceeding, let us stress that the value of the relative velocity sets the position of the Higgs resonance during DM annihilation. From eq. (2.2) it follows that the pole of the Higgs propagator is given by $s=m_{h}^{2}$; at zero relative velocity, therefore, the resonant annihilation occurs at $m_{\mathrm{S}}=m_{h} / 2=63 \mathrm{GeV}$, while in general it occurs at $m_{\mathrm{S}}^{2}=m_{h}^{2}(1-$ $\left.v_{\text {rel }}^{2} / 4\right) / 4$. Considering the annihilation in the early Universe - i.e. taking for definiteness $v_{\text {rel }}=1 / 2$ - the resonance occurs at $m_{\mathrm{S}} \simeq 61 \mathrm{GeV}$.

\section{$2.2 \quad$ LHC bound}

If $m_{\mathrm{S}}<m_{h} / 2$, the Higgs boson can decay into two DM particles resulting in the possibility to have a sizable invisible decay channel. In this case the invisible decay width of the Higgs is

$$
\Gamma_{\mathrm{h} \rightarrow \mathrm{SS}}\left(m_{\mathrm{S}}, \lambda_{\mathrm{S}}\right)=\frac{v^{2} \lambda_{\mathrm{S}}^{2}}{32 \pi m_{h}} \sqrt{1-\frac{4 m_{\mathrm{S}}^{2}}{m_{h}^{2}}} .
$$

The invisible branching ratio

$$
\mathrm{BR}_{\mathrm{inv}}\left(m_{\mathrm{S}}, \lambda_{\mathrm{S}}\right) \equiv \frac{\Gamma_{\mathrm{h} \rightarrow \mathrm{SS}}\left(m_{\mathrm{S}}, \lambda_{\mathrm{S}}\right)}{\Gamma_{\mathrm{h}}^{\mathrm{SM}}+\Gamma_{\mathrm{h} \rightarrow \mathrm{SS}}\left(m_{\mathrm{S}}, \lambda_{\mathrm{S}}\right)}
$$

is severely constrained by the current searches at the $\mathrm{LHC}[34,35]$, and $\mathrm{BR}_{\mathrm{inv}}>22 \%$ is excluded at $95 \%$ C.L. [36]. Using eq. (2.9) it is straightforward to translate this bound in the parameter space $\left(m_{\mathrm{S}}, \lambda_{\mathrm{S}}\right)$, and in section 4 we will include this constraint in our analysis. To give a quantitative idea of the size of the invisible branching ratio in eq. (2.9), notice that for the benchmark values $m_{\mathrm{S}}=20 \mathrm{GeV}, \lambda_{\mathrm{S}}=0.05$ we have $\mathrm{BR}_{\text {inv }} \simeq 73 \%$. As soon as the invisible decay channel is kinematically allowed, therefore, it can easily dominate over the SM final states even for relatively small values of the Higgs portal coupling. Notice that invisible width in eq. (2.8) is equal to zero for the resonant value $m_{\mathrm{S}}=m_{h} / 2$. Therefore, it will be impossible to test this particular region using the bound on the invisible branching ratio.

\subsection{Direct detection constraint}

The Higgs portal interaction, through the exchange of the Higgs boson in the t-channel, provides the possibility to have a non-zero spin-independent elastic cross-section of DM on nuclei.

Integrating out the Higgs in the limit of negligible exchanged momentum, it is possible to write the following effective interactions between DM and light quarks and gluons inside the nucleus

$$
\mathcal{L}_{\mathrm{S}}^{\mathrm{eff}}=\frac{\lambda_{\mathrm{S}}}{2 m_{h}^{2}} S^{2}\left(\sum_{q} m_{q} \bar{q} q-\frac{\alpha_{s}}{4 \pi} G^{2}\right),
$$

with $q=u, d, s$, and $G^{2}=G_{\mu \nu} G^{\mu \nu}$, where $G_{\mu \nu}$ is the gluon field strength. Using this effective interaction, it is straightforward to compute the spin-independent cross-section describing the elastic DM-nucleon scattering

$$
\sigma_{\mathrm{SI}}=\frac{\lambda_{\mathrm{S}}^{2} f_{\mathrm{N}}^{2}}{4 \pi} \frac{\mu_{\mathrm{S}}^{2} m_{\mathrm{N}}^{2}}{m_{h}^{4} m_{\mathrm{S}}^{2}}
$$


where $\mu_{\mathrm{S}} \equiv m_{\mathrm{N}} m_{\mathrm{S}} /\left(m_{\mathrm{N}}+m_{\mathrm{S}}\right)$ is the DM-nucleon reduced mass, $m_{\mathrm{N}}=0.946 \mathrm{GeV}$ is the nucleon mass, and $f_{\mathrm{N}}=0.303$ is the Higgs-nucleon coupling [29]. The LUX experiment has reported the strongest limit on $\sigma_{\mathrm{SI}}$ [37]. Using eq. (2.11) we translate this bound in the parameter space $\left(m_{\mathrm{S}}, \lambda_{\mathrm{S}}\right)$, and in section 4 we will include this constraint in our analysis.

Before proceeding, let us stress a simple but important point. The square of the momentum transferred in a typical DM-nucleus elastic scattering always satisfies the condition $-q^{2} \ll m_{h}^{2}$, with $q^{2}=-2 m_{\mathrm{Xe}} E_{\text {rec }}$ where the mass of a Xenon nucleus is $m_{\mathrm{Xe}}=121 \mathrm{GeV}$ and for the typical recoil energy one has $E_{\text {rec }} \sim$ few $\mathrm{keV}$. This simply implies that there is no resonant enhancement in elastic scatterings via the Higgs portal; as a consequence, the region with $m_{\mathrm{S}} \approx 63 \mathrm{GeV}$ where the model reproduces the correct relic abundance is beyond the present reach of direct detection experiments, and it will be covered only in the next future.

\section{Antiprotons: background vs. signal}

In this section we address the properties of the transport equation describing the propagation of cosmic rays in the Galaxy. In section 3.1, we discuss the background contribution, focusing our attention mostly on astrophysical background of protons and antiprotons. In section 3.2, we illustrate the strategy that we follow in order to extract our bound on the scalar Higgs portal model.

\subsection{Selection of propagation models}

Considering the total luminosity injected in the Milky Way galaxy via cosmic rays, most $(\sim 90 \%)$ of it consists of primary protons, $\sim 10 \%$ of helium nuclei, a further $\sim 1 \%$ of heavier nuclei, and $\sim 1 \%$ of free electrons. In full generality, the evolution of the cosmic-ray density in the Galaxy is described by the following transport equation

$$
\begin{aligned}
\frac{\partial N_{i}}{\partial t}= & \vec{\nabla} \cdot\left(D \vec{\nabla}-\overrightarrow{v_{c}}\right) N_{i}+\frac{\partial}{\partial p}\left(\dot{p}-\frac{p}{3} \vec{\nabla} \cdot \overrightarrow{v_{c}}\right) N_{i}+\frac{\partial}{\partial p} p^{2} D_{p p} \frac{\partial}{\partial p} \frac{N_{i}}{p^{2}}+Q_{i}(p, r, z) \\
& +\sum_{j>i} \beta n_{\text {gas }}(r, z) \sigma_{j i} N_{j}-\beta n_{\text {gas }} \sigma_{i}^{\text {in }}\left(E_{k}\right) N_{i},
\end{aligned}
$$

where $N_{i}=N_{i}(r, z, p, t)$ is the number density per total unit momentum of the $i$-th atomic species, $p$ is its momentum and $\beta$ is its velocity. The construction of a propagation model consists in solving eq. (3.1) with a certain boundary condition for all the cosmic-ray species; in this way one can compute - for a given distribution and energy spectrum of the sources - the spatial distribution and energy spectrum after propagation. Eq. (3.1) contains a number of free parameters to be determined. Let us discuss these parameters one by one.

- For each nucleus, the source distribution and the injection index $\gamma_{i}$ (or indices, if a break is considered). In eq. (3.1) these informations are encoded into the source term $Q_{i}(p, r, z)$. Considering the contribution of the astrophysical background, $Q_{i}(p, r, z)$ describes the distribution and injection spectrum of supernova remnants [38]. As far as the spectral index is concerned, we assume - following ref. [10] — the same spectral index $\gamma_{i}=\gamma$ for all the nuclear species. 
- The normalization and energy dependence of the diffusion coefficient, D. In eq. (3.1), we assume the following functional form

$$
D(\rho, r, z)=D_{0} \beta^{\eta} \mathrm{e}^{|z| / z_{t}}\left(\frac{\rho}{\rho_{0}}\right)^{\delta},
$$

where $\rho=p \beta /(Z e)$ is the rigidity of the nucleus of charge $Z, D_{0}$ is the absolute normalization at reference rigidity $\rho_{0}=3 \mathrm{GV}, \delta$ is the diffusion spectral index related to the turbulence of the interstellar medium, and $z_{t}$ is the scale height that controls the vertical spatial dependence, which is assumed to be exponential; the halo thickness $z_{h} \equiv 2 z_{t}$ is the height of the propagation halo where stochastic diffusion and re-acceleration take place. An additional parameter, $\eta$, controls the low-energy behavior of the diffusion coefficient.

- The Alfvén velocity, $v_{A}$. It parametrizes the efficiency of the stochastic re-acceleration mechanism. In eq. (3.1) it enters in the explicit expression of the diffusion coefficient in momentum space, $D_{p p}[10]$.

- The convective velocity, $\overrightarrow{v_{c}}$. It is the velocity of the convective wind, if present, that may contribute to the escape of cosmic rays from the Galactic plane. The convective velocity is zero in the Galactic plane and linearly increasing with the vertical distance $z$ from the Galactic plane.

- For each nucleus, the scattering cross-sections on the interstellar medium gas. The distribution of gas in the interstellar medium concentrates in the disk of the Galaxy. Its number density is denoted as $n_{\text {gas }}$, which is mainly constituted by atomic and molecular hydrogen and helium. There are two main effects. On the one hand, the $i$-th nucleus is generated by the nuclear species $j$ with cross-section $\sigma_{j i}$; on the other one, the $i$-th nucleus is destroyed by scattering on interstellar medium gas with total inelastic cross-section $\sigma_{i}^{\text {in }}$.

The propagation of cosmic rays can be simplified, if one takes into account only the high-energy region $(\gtrsim 10 \mathrm{GeV})$. In this regime, diffusion and energy losses play an important role while other effects, such as convection and re-acceleration, are negligible. However, following this approach one is forced to neglect all the cosmic ray data at low-energy; as a consequence, the ability of constraining DM models - especially with relative low mass - decreases dramatically. In order to take advantage of all the data set, the propagation equation needs to be solved numerically without these approximation. To achieve this result, we use the public code DRAGON [39, 40], and our procedure goes as follows.

Following ref [10], we start from five different benchmark propagation models: KRA, KOL, CON, THK and THN. These propagation models are characterized by different halo height $z_{t}$, slope of the diffusion coefficient $\delta$, spectral index $\gamma$, and gradient of the convection velocity $d v_{c} / d z$. We collect the corresponding values in table 1 . KRA, KOL and CON are characterized by the same halo height $\left(z_{t}=4 \mathrm{kpc}\right)$ but they describe differently turbulence effects and convection velocity. THK and THN, on the contrary, explore two extreme values 
for the halo height, namely $z_{t}=10 \mathrm{kpc}$ and $z_{t}=0.5 \mathrm{kpc}$. Using these five benchmark propagation models, the intent is to capture a wide range of astrophysical uncertainties. For a more detailed discussion we refer the interest reader to ref. [10].

The second step in our analysis is to use the Boron-over-Carbon (B/C hereafter) data in order to determine - for each one of the propagation setups defined before - the remaining phenomenological parameters in eq. (3.1). The B/C data employed in our analysis come from the HEAO3 [41], ACE [42, 43],CREAM [44] ATIC [45] and CRN [46] experiments. In table 1 , we complete the definition of the five benchmark propagation models by minimizing the $\chi^{2}$ against $\mathrm{B} / \mathrm{C}$ data; in this way we obtain, as output of the fitting procedure, $D_{0}, \eta$ and the Alfvén velocity $v_{A}$. The $\mathrm{B} / \mathrm{C}$ data with energy larger than $0.5 \mathrm{GeV}$ are considered, and - in the context of the force field approximation - the value of the solar modulation potential is treated as follows. First, we note that CREAM, ATIC and CRN data are not affected by solar modulation, since they fall in the high-energy region (see figure 2 below); as far as these experiments are concerned, therefore, we directly use the local interstellar spectrum without including solar modulation in our fit. On the contrary, HEAO3 data cover also the low-energy region where the role of solar modulation can not be neglected. To get around this problem, we decided to use a fixed value for the solar modulation potential, namely $\Phi=0.55 \mathrm{GV}$. The reason for this choice is twofold. On a more practical level, this simplified assumption allows us to increase the speed of our numerical code. On the other hand, the fit of the $\mathrm{B} / \mathrm{C}$ data is dominated by the high-energy region, and as a consequence the impact of different values for the solar modulation potential on the $\chi^{2}$ function is only marginal; we explicitly checked that different choices for $\Phi$ do not lead to appreciable deviation in the output parameters. The situation is completely different considering the antiproton-to-proton data where the low-energy part of the measured spectrum is crucial in order to obtain reliable bounds. As we shall see in the following, in fact, in this case we will fit the solar modulation potential against the experimental data.

The reason why we chose to define our benchmark propagation models focusing exclusively on the $\mathrm{B} / \mathrm{C}$ data is that antiproton flux may originate both from astrophysical and exotic sources, while Boron and Carbon are usually generated only by astrophysical processes. Moreover, $\mathrm{B} / \mathrm{C}$ represents the ratio between stable secondary cosmic-ray flux divided by the corresponding primary cosmic-ray flux, which is exactly the same as $\bar{p} / p$ that we will use in the next step of our analysis. In the left panel of figure 2 we show the best-fit value for the $\mathrm{B} / \mathrm{C}$ flux for the five propagation models in table 1 . Solid lines are obtained using $\Phi=0 \mathrm{GV}$; for completeness, we also show that it is possible to fit the low-energy data of the ACE experiment with a proper tuning of the solar modulation potential (dashed lines).

Finally, we can use the five propagation models in table 1 to compute the background contribution to the $\bar{p} / p$ flux. The choice of the $\bar{p} / p$ ratio is made with the purpose of decreasing the systematic uncertainties that come from the comparison of data taken by different experiments. As far as the antiproton flux is concerned, for instance, various experiments can have different absolute flux due to different energy calibrations, which we want to avoid. Using data describing the $\bar{p} / p$ flux, on the contrary, we can safely combine different datasets. The $\bar{p} / p$ data employed in our analysis come from the BESS [47, 48], 


\begin{tabular}{|c|c|c|c|c|c|c|c|c|c|c|c|}
\hline Model & $\begin{array}{c}z_{t} \\
(\mathrm{kpc})\end{array}$ & $\delta$ & $\gamma$ & $\begin{array}{c}d v_{c} / d z \\
\left(\mathrm{~km} \mathrm{~s}^{-1} \mathrm{kpc}^{-1}\right)\end{array}$ & $\begin{array}{c}D_{0} \\
\left(10^{28} \mathrm{~cm}^{2} \mathrm{~s}^{-1}\right)\end{array}$ & $\eta$ & $\begin{array}{c}v_{A} \\
\left(\mathrm{~km} \mathrm{~s}^{-1}\right)\end{array}$ & $\begin{array}{c}\Phi \\
(\mathrm{GV})\end{array}$ & $\chi_{\mathrm{B} / \mathrm{C}}^{2}$ & $\chi_{\bar{p} / p}^{2}$ & $\chi_{\bar{p} / p, \text { PAMELA }}^{2}$ \\
\hline KRA & 4 & 0.50 & 2.35 & 0 & 2.68 & -0.384 & 21.07 & 0.950 & 0.95 & 1.26 & 1.08 \\
\hline THN & 0.5 & 0.50 & 2.35 & 0 & 0.32 & -0.600 & 17.87 & 0.950 & 0.88 & 1.41 & 1.26 \\
\hline THK & 10 & 0.50 & 2.35 & 0 & 4.45 & -0.332 & 19.91 & 0.950 & 0.98 & 1.24 & 1.08 \\
\hline KOL & 4 & 0.33 & $1.78 / 2.45$ & 0 & 4.45 & 1.00 & 40.00 & 0.673 & 0.57 & 1.11 & 0.93 \\
\hline CON & 4 & 0.60 & $1.93 / 2.35$ & 50 & 0.99 & 0.786 & 40.00 & 0.19 & 0.58 & 1.00 & 0.67 \\
\hline
\end{tabular}

Table 1. Phenomenological parameters describing the five benchmark propagation models (first column) used in our analysis. In the next four columns of the table we collect the values of halo height $z_{t}$, slope of the diffusion coefficient $\delta$, spectral index $\gamma$, and gradient of the convective velocity $d v_{c} / d z$; these values are kept fixed, and define - for each propagation models - the corresponding properties of the diffusion-loss equation (3.1). The normalization of the diffusion coefficient $D_{0}$, the low-energy parameter $\eta$ and the Alfvén velocity $v_{A}$ are obtained via a $\chi^{2}$ fit of the $\mathrm{B} / \mathrm{C}$ data from the HEAO3 [41], CREAM [44], ATIC [45] and CRN [46] experiments; in addition, we show the corresponding minimum $\chi_{\mathrm{B} / \mathrm{C}}^{2}$ (divided by the number of degrees of freedom). Using the propagation models so defined, we compute the background contribution to the $\bar{p} / p$ flux, and we fit the solar modulation potential $\Phi$ against data from the BESS, CAPRICE and PAMELA experiments. We show in the last two columns the corresponding values of $\chi_{\bar{p} / p}^{2}$ (against, respectively, the full dataset and the subset of PAMELA data). The reported values for the solar modulation potential $\Phi$ refer to the fit of the PAMELA data only.

CAPRICE [49] and PAMELA [50] experiments. At this stage, the only free parameter is the value of solar modulation; in principle, since different experiments operated at different time, we can use three independent values of $\Phi$ - one for each experiment - in order to fit the data, and in table 1 we show the result of the corresponding $\chi^{2}$ fit (see caption for details). ${ }^{3}$

Once the astrophysical background has been fixed, we are now in the position to discuss the contribution from DM annihilation in the Higgs portal model.

\subsection{Antiproton bound on the scalar Higgs portal model}

We now move to discuss the computation of the antiproton flux originated from DM annihilation in the context of the Higgs portal model.

We solve eq. (3.1) using as a source term

$$
Q_{\bar{p}}^{\mathrm{DM}}(p, r, z)=\left.\frac{1}{2}\left[\frac{\rho_{\mathrm{DM}}(r, z)}{m_{\mathrm{S}}}\right]^{2} \frac{d N}{d E}\right|_{\bar{p}}\left\langle\sigma v_{\mathrm{rel}}\right\rangle_{0}
$$

where $\rho_{\mathrm{DM}}$ is the DM density profile, $d N /\left.d E\right|_{\bar{p}}$ the antiproton emission spectrum, i.e. the number of antiprotons per each annihilation, and $\left\langle\sigma v_{\text {rel }}\right\rangle_{0}$ is the thermally averaged annihilation cross-section times relative velocity evaluated at the present epoch. The solution of eq. (3.1) allows us to compute - as a function of DM mass and portal coupling,

\footnotetext{
${ }^{3}$ We vary the solar modulation potential in the range $\Phi \in[0,0.95]$ GV. For completeness, we report here the best-fit values (in GV) for the solar modulation potential related to the fit of both BESS and CAPRICE data. BESS: $\Phi_{\mathrm{KRA}}=0.76, \Phi_{\mathrm{THN}}=0.79, \Phi_{\mathrm{THK}}=0.73, \Phi_{\mathrm{KOL}}=0.48, \Phi_{\mathrm{CON}}=0.11$. CAPRICE: $\Phi_{\mathrm{KRA}}=0.87, \Phi_{\mathrm{THN}}=0.90, \Phi_{\mathrm{THK}}=0.85, \Phi_{\mathrm{KOL}}=0.61, \Phi_{\mathrm{CON}}=0.22$.
} 

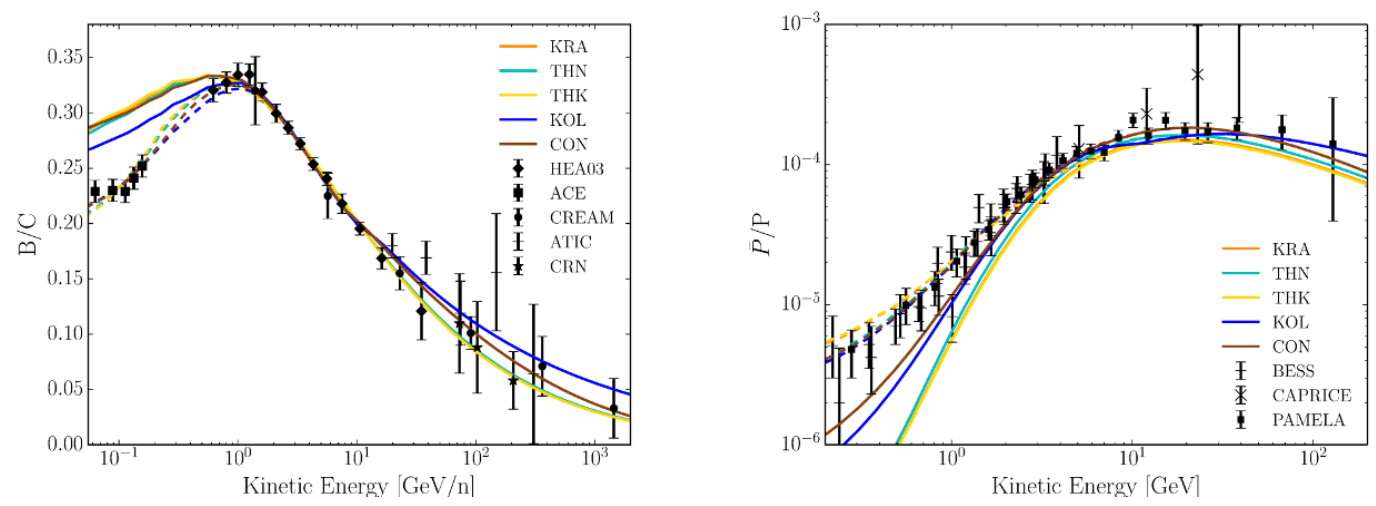

Figure 2. Fit of the B/C (left panel) and $\bar{p} / p$ (right panel) data using the five propagation models defined in table 1. Solid lines correspond to the background model prediction without considering solar modulations, while dotted lines correspond to the prediction with solar modulations (see text for details).

and at the location of the Earth - the antiproton flux originated from DM annihilation, $\phi_{\bar{p}}^{\mathrm{DM}}\left(m_{\mathrm{S}}, \lambda_{\mathrm{S}}\right)$. Next, we compute the local $\bar{p} / p$ flux by combining DM contribution and astrophysical background, $\phi_{\bar{p} / p}\left(m_{\mathrm{S}}, \lambda_{\mathrm{S}}\right)=\left[\phi_{\bar{p}}^{\mathrm{BG}}+\phi_{\bar{p}}^{\mathrm{DM}}\left(m_{\mathrm{S}}, \lambda_{\mathrm{S}}\right)\right] / \phi_{p}^{\mathrm{BG}}$. Finally, by means of a $\chi^{2}$ fit of the $\bar{p} / p$ data, we extract a bound on the parameter space of the scalar Higgs portal model.

We will discuss in section 4.2 the impact of different DM density profiles on the results of our analysis. In the computation of the antiproton emission spectrum, we included - consistently with the computation of the relic density - the three-body final states consisting of one on-shell and one off-shell electroweak gauge bosons. Following ref. [51], we made use of the PYTHIA 8.1 event generator $[52,53]$ to extract these energy spectra.

\section{Results}

Following the approach outlined in section 3, we derived the bound on the parameter space of the scalar Higgs portal model by analyzing the antiproton-to-proton ratio data, and in this section we present and discuss our main results. We show the bound as a $3-\sigma$ exclusion line in the planes $\left(m_{\mathrm{S}}, \lambda_{\mathrm{S}}\right)$ and $\left(m_{\mathrm{S}},\left\langle\sigma v_{\text {rel }}\right\rangle_{0}\right)$. In both cases we compare the antiproton bound with the region that reproduces the correct amount of relic abundance, according to the result of the numerical analysis outlined in section 2.1. In addition, we superimpose the constraints obtained considering the invisible Higgs decay width and the spin-independent DM-nucleon elastic cross-section as described, respectively, in sections 2.2 and 2.3.

In section 4.1 we analyze the impact of different propagation models while in section 4.2 we discuss the impact of different DM density profiles.

\subsection{On the impact of different propagation models}

In this section, we study the antiproton bound on varying the propagation model, according to table 1 . We show our results in figure 3 and in figure 4 where, for definiteness, we use the NFW DM density profile [54] (see figure 6, eq. (4.1), and table 2 below). 

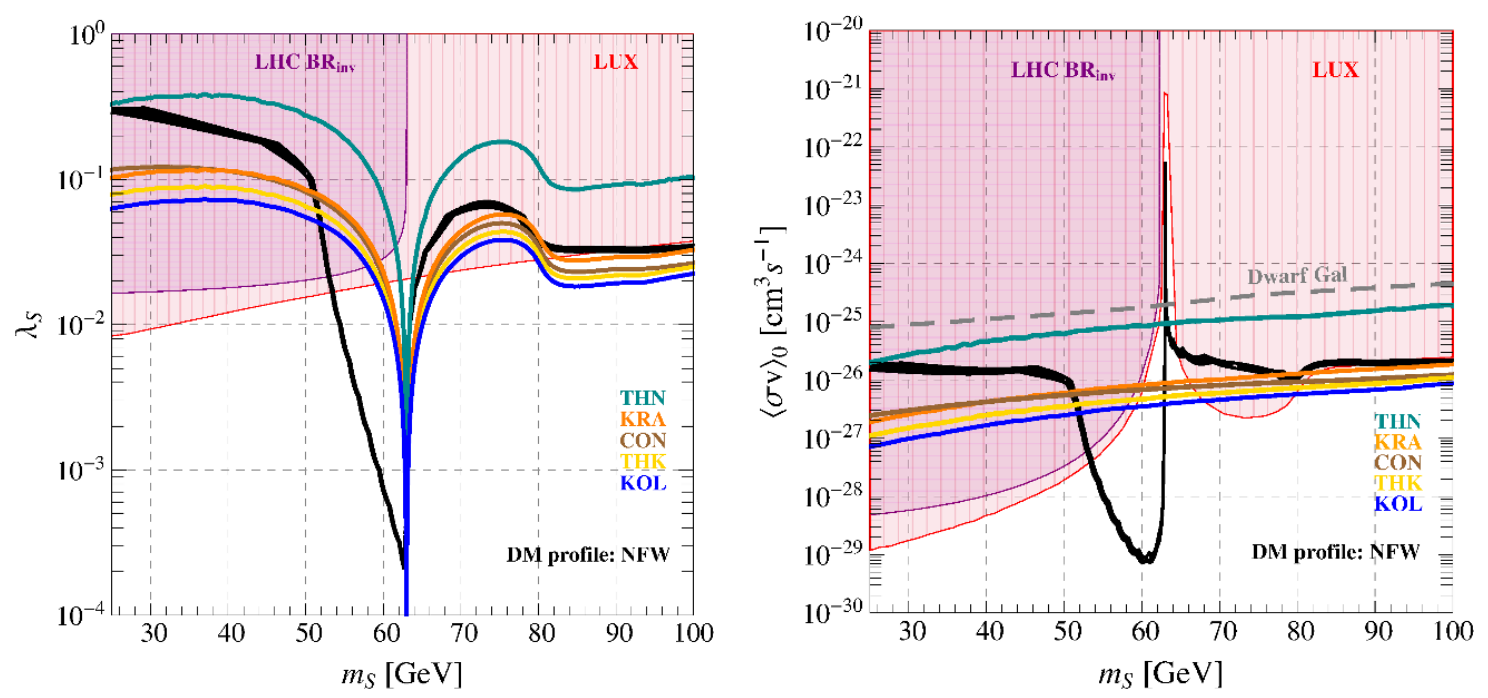

Figure 3. Bounds on the scalar Higgs portal model in the low-mass region. The black strip represents the $5 \sigma$ band reproducing the correct amount of relic density as measured by the Planck experiment. We show the region excluded at $95 \%$ C.L. by the LHC considering the invisible branching ratio of the Higgs (purple region, horizontal meshes) and the region excluded at 95\% C.L. by the LUX experiment considering direct detection of DM (red region, vertical meshes). In addition, we show the constraints obtained from the antiproton data considering as a benchmark model for the DM density the NFW profile. Different propagation models are displayed with different colors [from bottom (more stringent) to top (less stringent): KOL (blue), THK (yellow), KRA (orange), CON (brown), THN (dark cyan); see text for details]. The region above each one of these curves is excluded. The dashed gray line represents the bound placed by the Fermi-LAT experiment using the gamma-ray data from dwarf galaxies (see text). Left panel: bounds on the parameter space $\left(m_{\mathrm{S}}, \lambda_{\mathrm{S}}\right)$. Right panel: bounds on the thermally averaged annihilation cross-section times relative velocity at zero temperature.

Let us start the discussion with some general comments. The region that reproduces the correct value of relic density is represented by a black strip, while the regions excluded by the LHC and LUX experiments are shaded, respectively, in purple and red. In figure 3 we focus on small values for the DM mass, i.e. $m_{\mathrm{S}} \in[25,100] \mathrm{GeV}$, in order to emphasize the role of the antiproton bound in the region close to the Higgs resonance. In the left panel of figure 3 we show our results in the parameter space $\left(m_{\mathrm{S}}, \lambda_{\mathrm{S}}\right)$; considering the black strip that reproduces the correct value of relic abundance, the resonant region is immediately recognizable because of the usual funnel-shaped form. As already discussed in section 2.1, this region extends also for values of the DM mass smaller than $m_{h} / 2=63 \mathrm{GeV}$ as a consequence of thermal effects during the freeze-out epoch, and this feature clearly emerges in the plot from the result of our numerical analysis; moreover, notice that both the bound on the invisible Higgs decay width and the spin-independent DM-nucleon cross-section can not rule out this region because of the kinematical reasons discussed in sections 2.2, 2.3. In the right panel of figure 3, we translate our analysis in the plane $\left(m_{\mathrm{S}},\left\langle\sigma v_{\text {rel }}\right\rangle_{0}\right)$. Away from the resonance the value of $\left\langle\sigma v_{\text {rel }}\right\rangle_{0}$ that reproduces the observed relic abundance is close to the usual WIMP-miracle cross-section $\left\langle\sigma v_{\text {rel }}\right\rangle_{0} \approx 2 \times 10^{-26} \mathrm{~cm}^{3} \mathrm{~s}^{-1}$. Close to the 

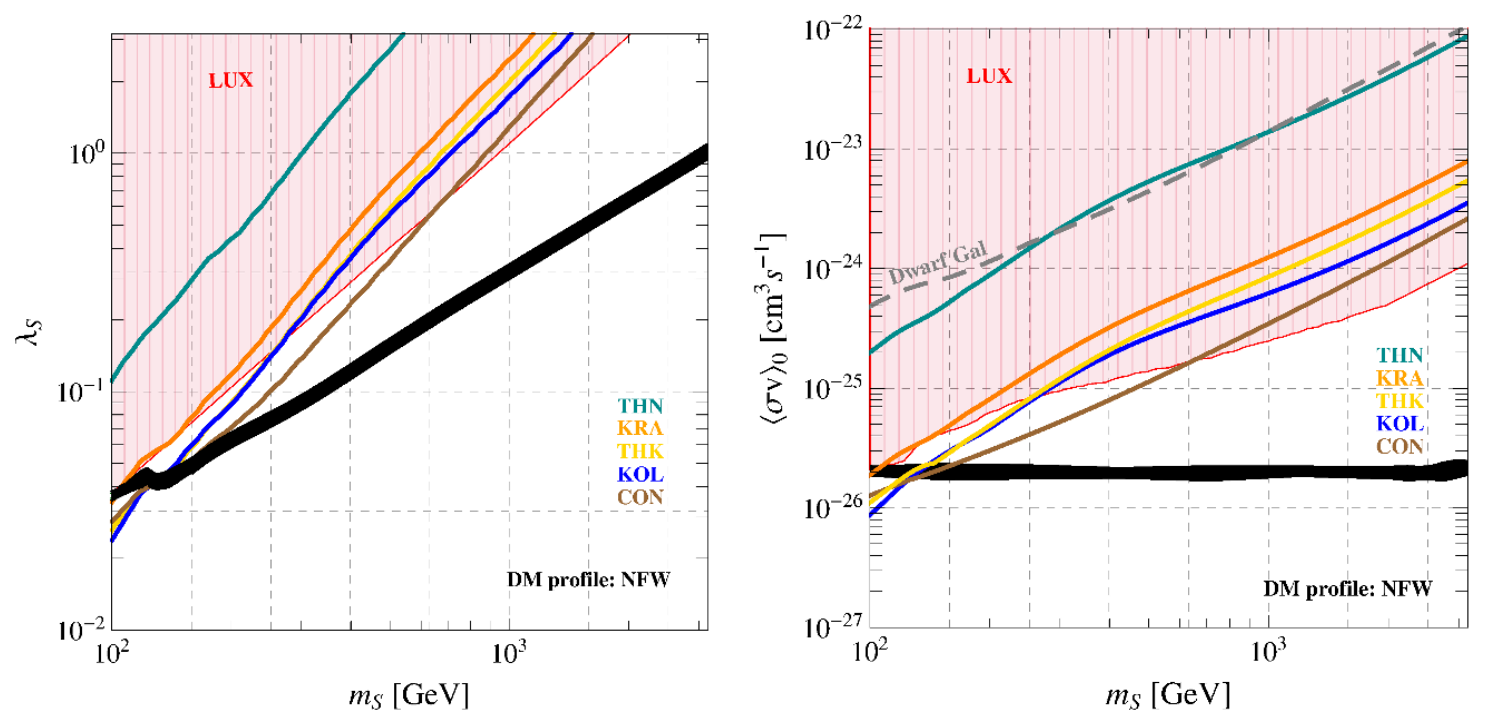

Figure 4. The same as in figure 3, but for large values of the DM mass.

resonance, on the contrary, this value is distorted by the presence of the aforementioned thermal effects that move the position of the resonance during the freeze-out epoch. In particular, we find that for $50 \lesssim m_{\mathrm{S}} \lesssim m_{h} / 2$ the thermally averaged annihilation crosssection times relative velocity today can be as small as $\left\langle\sigma v_{\text {rel }}\right\rangle_{0} \approx 10^{-29} \mathrm{~cm}^{3} \mathrm{~s}^{-1}$, while for $m_{\mathrm{S}} \approx m_{h} / 2$ we have $\left\langle\sigma v_{\text {rel }}\right\rangle_{0} \approx 10^{-22} \mathrm{~cm}^{3} \mathrm{~s}^{-1}$. In figure 4 we focus on large values for the DM mass, i.e. $m_{\mathrm{S}} \in[100,3000] \mathrm{GeV}$. As in figure 3, we show the constraints placed by our phenomenological analysis in the plane $\left(m_{\mathrm{S}}, \lambda_{\mathrm{S}}\right)$, left panel, and $\left(m_{\mathrm{S}},\left\langle\sigma v_{\text {rel }}\right\rangle_{0}\right)$, right panel.

In figure 3 and figure 4 we show the antiproton bound that corresponds to the five propagation models defined in section 3 , and we use the same color code introduced in table 1 . The results of our analysis point towards the following remarks.

- On a general ground, we find that the KOL, THK, CON and KRA propagation models give similar bound, while the THN propagation model places the weaker constraint. In greater detail, in the low mass region the KOL model, characterized by a strong re-acceleration, gives the strongest constraint. On the contrary in the high mass region the CON model, characterized by a strong convective wind, provides the most stringent bound. The presence of strong convective effects, in fact, hardens the antiproton flux thus leading to stronger constraints on heavier DM models [10]. It is worth noticing that the THN model, based on a thin diffusion zone, is disfavored by recent studies on synchrotron emission, radio maps and low energy positron spectrum [55].

- In the low mass region the antiproton bound is competitive with the bound obtained from direct detection and invisible Higgs decay width. In particular, we find that the bound from antiproton is the only one able to rule out the resonant region with $m_{\mathrm{S}} \approx m_{h} / 2$. Let us stress once again that this specific value for the DM mass would be otherwise inaccessible. On the one hand, in fact, the invisible Higgs branching 
ratio goes to zero moving towards the kinematical threshold $m_{\mathrm{S}}=m_{h} / 2$; on the other one, the square of the momentum transferred in a typical DM-nucleus elastic scattering always satisfies the condition $-q^{2} \ll m_{h}^{2}$, with $q^{2}=-2 m_{\mathrm{Xe}} E_{\text {rec }}$ where the mass of a Xenon nucleus is $m_{\mathrm{Xe}}=121 \mathrm{GeV}$ and for the typical recoil energy one has $E_{\text {rec }} \sim$ few $\mathrm{keV}$.

- In the high mass region the antiproton bound obtained using the KOL, THK, CON and KRA propagation models is competitive with the exclusion curve traced by the LUX experiment. In particular, as clear from the right panel of figure 4, using the KOL, THK and CON propagation models it is possible to probe the thermal crosssection up to $m_{\mathrm{S}} \approx 160 \mathrm{GeV}$.

- For comparison, we show in the right panel of figures 3, 4 the 95\% C.L. exclusion curve obtained considering the measurement of the gamma-ray flux from the dwarf spheroidal satellite galaxies of the Milky Way [56]. ${ }^{4}$ These dwarf galaxies are some of the most DM-dominated objects known, and - because of their proximity, high DM content, and lack of astrophysical backgrounds - they are usually considered to be the most promising targets for the indirect detection of DM via gamma rays. For simplicity, in our analysis we used only the data from the Draco dwarf spheroidal galaxy since it gives the strongest constraint. Let us now describe in more detail our approach. In order to use the result of ref. [56], first we computed the gamma-ray flux from the Draco dwarf spheroidal galaxy in the Higgs portal model under scrutiny, combining all the different annihilation channels including three-body final states. Then, for each value of the DM mass, we compared the gamma-ray flux previously obtained with the $95 \%$ C.L. exclusion limit in each of the 24 energy bins analyzed in figure 2 of ref. [56]. Finally, we extracted the bound on the cross-section from the energy bin that provides the strongest constraint. We find that, both in the low- and high-mass regions, the bound from antiproton that we obtain using the KOL, THK, CON and KRA propagation models is more than one order of magnitude stronger than the bound obtained from the analysis of the gamma-ray flux measured from the Draco dwarf spheroidal galaxy. Needless to say, a more detailed analysis would require to include all the 25 dwarf spheroidal galaxies studied in ref. [56] together with a more careful investigation of the systematic errors involved. This task goes well beyond the purpose of the simple estimation that we derived in this work, and will be left for future investigation.

In conclusion, we have found that the antiproton bound provides a strong constraint on the parameter space of the scalar Higgs portal model introduced in section 2. Remarkably, the constraining power of the antiproton data is comparable to the exclusion curves placed by the LHC and LUX experiments in particular for $m_{\mathrm{S}} \gtrsim 50 \mathrm{GeV}$. Most importantly, the antiproton bound is the only one able to rule out the Higgs resonant region for $m_{\mathrm{S}} \approx 63 \mathrm{GeV}$. This conclusion does not strongly depend on the model used to describe the

\footnotetext{
${ }^{4}$ There is a new preliminary result of the dwarf galaxies presented by the Fermi-LAT group at the 2014 Fermi Symposium [57].
} 

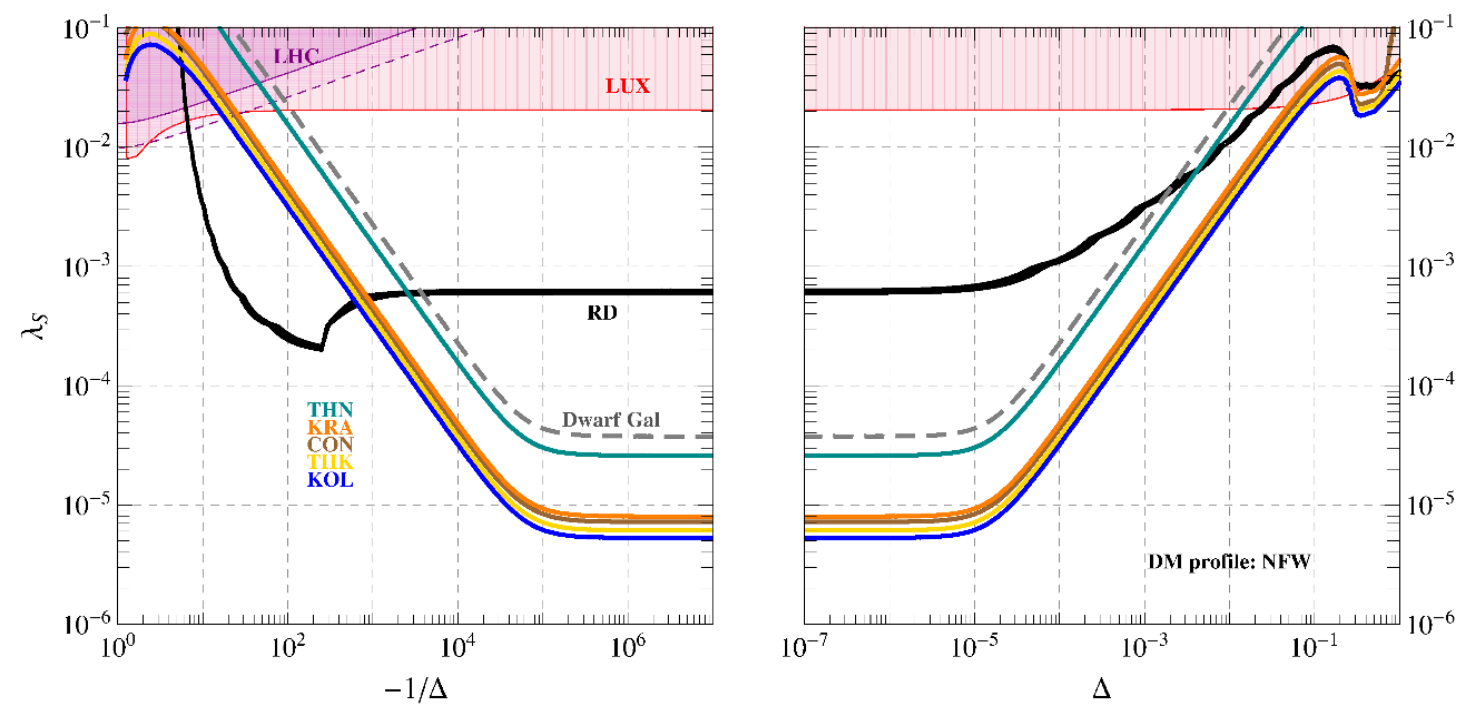

Figure 5. The same as in figure 3, but with a special focus on the Higgs resonance.

dynamics underlying the propagation of charged particle in the Galaxy; in particular, we have shown that the KOL, THK, CON and KRA propagation setups give, in magnitude, similar bounds. In order to stress this point we show in figure 5, following ref. [58], a zoom on the Higgs resonant region. We introduce the variable $\Delta \equiv\left(2 m_{\mathrm{S}}-m_{h}\right) / m_{h}$, and we present our constraints in the plane $\left(\Delta, \lambda_{\mathrm{S}}\right)$. From this point of view, it is clear that the bound we get from the antiproton data is by far the most stringent if compared with LHC and LUX results. The only region left unconstrained by the antiproton bound is the small mass window with $10 \lesssim-1 / \Delta \lesssim 10^{3}$ which corresponds to $56.7 \lesssim m_{\mathrm{S}} \lesssim 62.9 \mathrm{GeV}$ with $10^{-4} \lesssim \lambda_{\mathrm{S}} \lesssim 10^{-2}$. In this region the position of the Higgs resonance is subject to thermal effects; as previously discussed, in this small mass window the resonant annihilation cross-section reproducing in the early Universe the correct relic abundance corresponds to an off-resonant value in today's annihilations in the Galactic halo.

In this section we extracted the antiproton bound using the standard NFW profile in order to describe the density distribution of DM in the Galaxy. In the next section, we will discuss the impact of different DM halo profiles.

\subsection{On the impact of different DM density profiles}

In this section we explore the impact of different DM density profile on the analysis of the antiproton data. In addition to the NFW profile [54] already used in section 4.1, we repeat our analysis using the Einasto [59,60] and the Isothermal profile [61]. The former - similar to the NFW profile and characterized by a DM density distribution peaked towards the Galactic center - is favored by the latest standard numerical simulations $[62,63]$ while the latter - characterized by a constant core — seems to be in agreement with the numerical simulations that include baryons, because of large exchange of angular momentum between the gas and DM particles [64]. We show these three DM density distributions in figure 6 and eqs. (4.1), (4.2), (4.3), while in table 2 we collect the numerical values of the parameters 


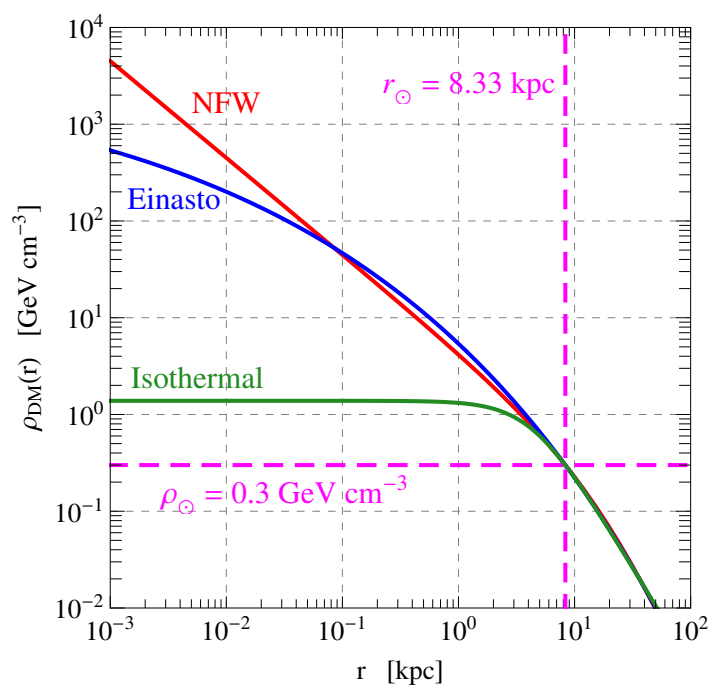

Figure 6. DM density distributions in eqs. (4.1), (4.2), (4.3).

\begin{tabular}{|c|c|c|}
\hline DM halo & $r_{s}[\mathrm{kpc}]$ & $\rho_{s}\left[\mathrm{GeVcm}^{-3}\right]$ \\
\hline NFW & 24.42 & 0.184 \\
\hline Ein & 28.44 & 0.033 \\
\hline Iso & 4.38 & 1.385 \\
\hline
\end{tabular}

Table 2. Parameters defining the DM density distributions in figure 6. For the Einasto profile, $\alpha=0.17$.

that enter in their definitions.

$$
\begin{aligned}
\rho_{\mathrm{NFW}}(r) & =\rho_{s} \frac{r_{s}}{r}\left(1+\frac{r}{r_{s}}\right)^{-2}, \\
\rho_{\operatorname{Ein}}(r) & =\rho_{s} e^{\left\{-\frac{2}{\alpha}\left[\left(\frac{r}{r_{s}}\right)^{\alpha}-1\right]\right\}}, \\
\rho_{\text {Iso }}(r) & =\frac{\rho_{s}}{1+\left(\frac{r}{r_{s}}\right)^{2}} .
\end{aligned}
$$

We show our results in figure 7 , for the low mass region, and in figure 8 , for the high mass region. In both cases we focus on the plane $\left(m_{\mathrm{S}},\left\langle\sigma v_{\text {rel }}\right\rangle\right)$.

Let us start our discussion pointing out an important argument to keep in mind for the rest of the section. As a rule of thumb, one would expect that the antiproton flux from DM annihilation is larger for profiles in which the DM density is enhanced towards the Galactic center while is smaller for density distribution described by an isothermal sphere; as a consequence, one would naïvely guess that a common feature of the analysis is that the antiproton bound is always more (less) stringent for the NFW (Isothermal) profile. In general, however, this conclusion turns out to be partially incorrect. What really matters, in fact, is not the value of DM density at the Galactic center but at the position where the antiprotons - whose flux is measured on Earth — are generated. As 

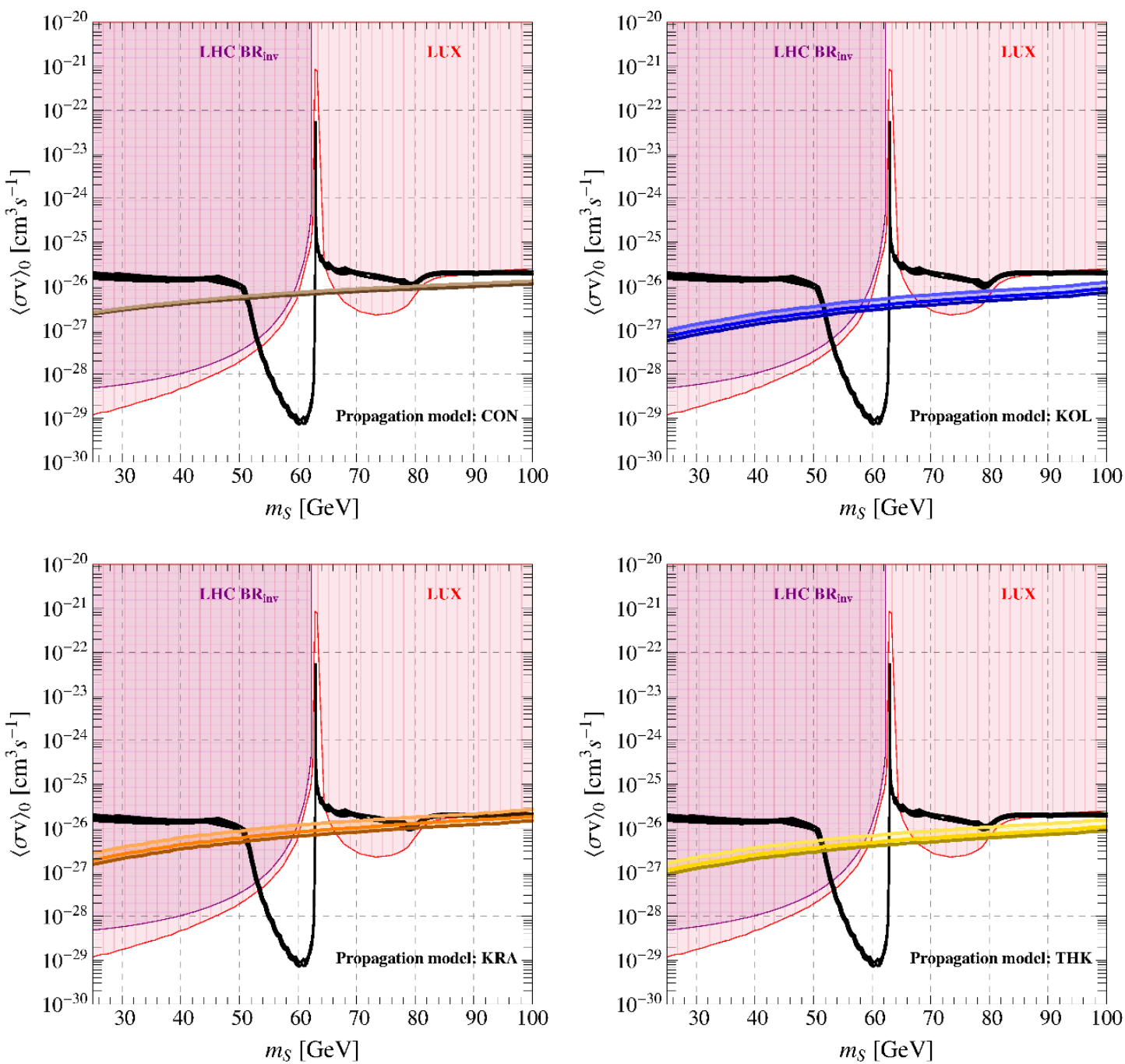

Figure 7. Antiproton bounds on the thermally averaged annihilation cross-section times relative velocity for different DM density distributions, namely the Einasto, NFW and Isothermal profiles. The most (less) stringent bound corresponds to the Einasto (Isothermal) profile. For comparison, we show the impact of different profiles for the four propagation models CON, KOL, THK, KRA (from top left, clockwise). The color code - as well as the other bounds from the LHC and LUX experiments - follows figure 3. The bound obtained for the THN propagation model does not depend on the DM density distribution, see text for details.

one can easily imagine, further insights on this issue are strongly linked to the assumed propagation model, and our analysis points towards the following results.

- We find that the antiproton bound obtained using the THN model, based on a very thin diffusion zone, does not significantly depend on the DM density profile, and we do not show the corresponding plot in figures 7), (8. In the THN model, in fact, the antiproton flux from DM annihilation is dominated by local contributions where the three profiles are equivalent [10]. 

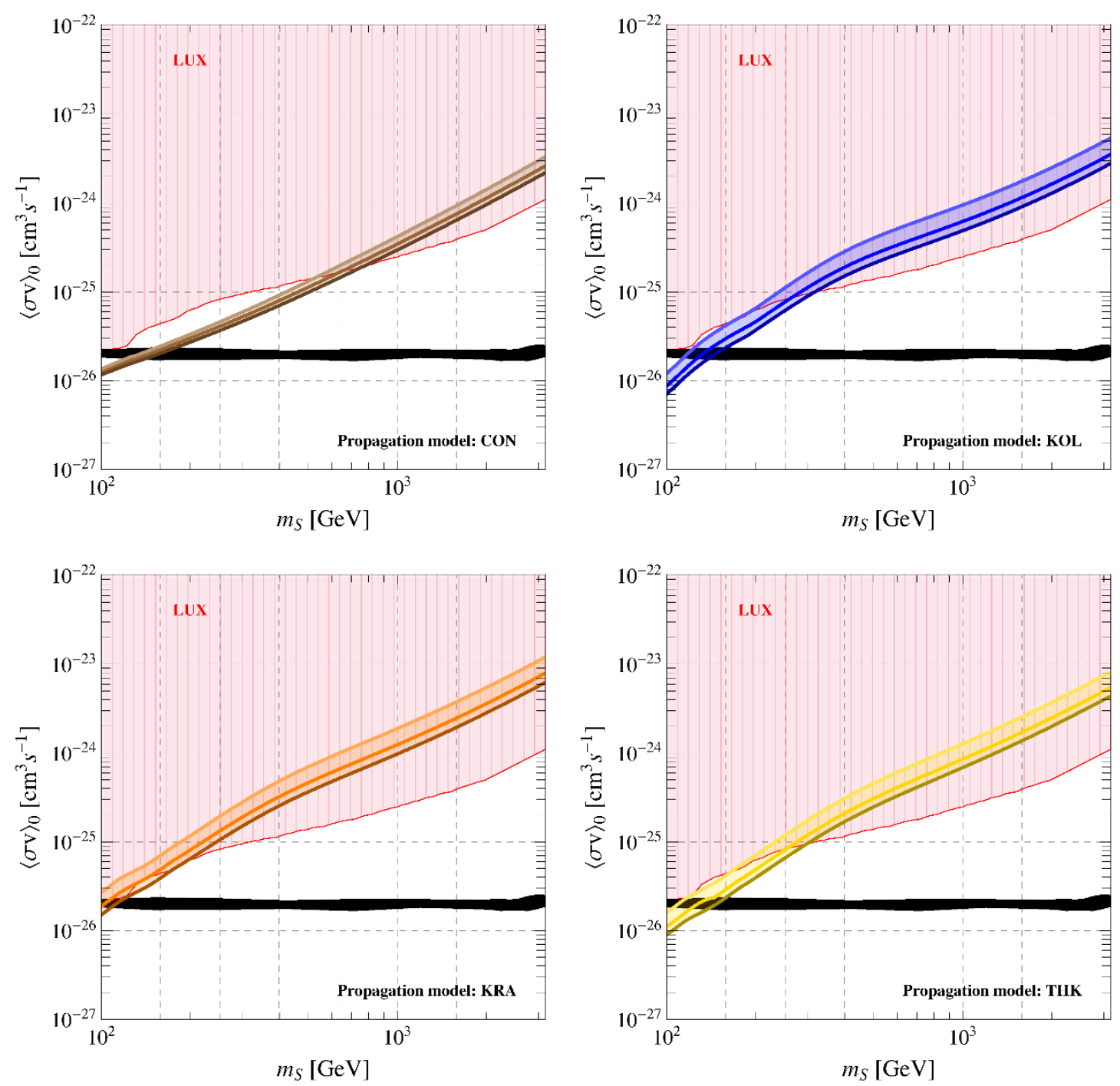

Figure 8. The same as in figure 7, but for large values of the DM mass.

- As far as the antiproton bound obtained assuming the CON propagation model is concerned, we find that also in this case the impact of different DM density distribution is moderately negligible, as shown in the upper-left panel of figures 7, 8. As already observed in ref. [10], therefore, we argue that the uncertainty related to the DM distribution towards the Galactic center has a negligible effect in the CON model in which the antiproton flux from DM annihilation is dominated by local contributions.

- The impact of different DM density distribution is relevant considering the antiproton bound obtained using the KOL, THK and KRA propagation models. In these models, therefore, a large contribution on the antiproton flux from DM annihilation comes from non-local regions pointing towards the Galactic center where the three profiles present sizable differences, being more or less peaked. In greater detail, we find that the Isothermal (Einasto) profile gives the weaker (stronger) constraint; moreover, 
comparing with the NFW case, the bound obtained assuming the Isothermal density distribution has the largest deviation, while the Einasto density distribution gives a similar result. Comparing the three profiles, as done in figure 6 , we notice that in the region $r \gtrsim 0.5 \mathrm{kpc}$ - being $\mathrm{r}$ the radial distance from the Galactic center - the density distribution in both the Einasto and the NFW profiles are significantly larger than the Isothermal case; moreover, in this region the Einasto density distribution is larger if compared with the NFW one, thus reflecting the hierarchy observed in the exclusion curves. On the contrary, for $r \lesssim 0.5 \mathrm{kpc}$, the density distribution in the NFW case is larger w.r.t. the Einasto profile. All in all, we argue that in the KOL, THK and KRA propagation model the antiproton flux from DM annihilation is dominated by regions close to the Galactic center, with $0.5 \lesssim r \lesssim r_{\odot} \mathrm{kpc}$. In order to strengthen this argument, we show in figure 9 the local antiproton flux coming from DM annihilation and, in the inset plot, the relative contribution from a region enclosed within $1 \mathrm{kpc}$ from the Galactic center. For definiteness, we consider $\left\langle\sigma v_{\text {rel }}\right\rangle_{0}=3 \times 10^{-26} \mathrm{~cm}^{3} \mathrm{~s}^{-1}$ and DM annihilation into $\bar{b} b\left(W^{+} W^{-}\right)$for $m_{\mathrm{S}}=70 \mathrm{GeV}$ $\left(m_{\mathrm{S}}=700 \mathrm{GeV}\right) .{ }^{5}$ From this plot it is clear that for all the propagation models the contribution to the total antiproton flux from the inner Galactic region is at most 20\%. Moreover, as expected, the THN and CON propagation models receive a negligible contribution from the region with $r<1 \mathrm{kpc}$. The thin height of the diffusion zone and the presence of strong convective wind efficiently remove a large fraction of the antiprotons originated towards the center of the Galaxy increasing their escape probability.

In conclusion, we have shown that the role of the DM density distribution in the Galaxy plays only a relatively marginal role in our analysis, and the astrophysical uncertainties affecting the limits on the scalar Higgs portal model that one can obtain using the antiproton data are mostly dominated by the details of the propagation model. ${ }^{6}$ In this regards, the antiproton data that will be released by the AMS-02 experiment will play a crucial role in order to improve the current sensitivity. In the next section, therefore, we will briefly discuss future perspectives.

\footnotetext{
${ }^{5}$ Without solar modulation, as plotted in the left panel of figure 9, the CON and THN models have similar flux at given DM mass $\left(m_{S}=70 \mathrm{GeV}\right)$ and cross section. However, their antiproton bounds are different as shown in figure 3. The reason is that the CON model gives harder cosmic-ray spectrum, which asks for smaller value of solar modulation. Taking properly into account the different values of solar modulation, the THN model has looser bound than the CON one.

${ }^{6}$ We remind that in this paper we do not consider additional uncertainties that could affect the computation of the antiproton flux both from DM annihilation and cosmic rays. Strong outflows from the Galactic center, for instance, can carry away a lot of annihilation products thus weakening the correlation between the $\bar{p}$ locally observed at Earth and the ones produced by DM annihilation; a more detailed analysis of astrophysical uncertainties can be found in ref. [10]. Notice, moreover, that in our analysis we extract the bound on DM using a fixed value for the solar modulation potential (the one obtained from the best-fit of the background contribution, see table 1). A different approach is based on the possibility to treat this variable as a nuisance parameter in the fit, thus leading to slightly weaker constraints; a more detailed analysis along this line can be found in refs. [9, 19]. Finally, there is an additional source of uncertainty related to the nuclear cross-sections describing the production of secondary $\bar{p}$. If introduced, this additional uncertainty will weaken the bound $[9,20]$.
} 

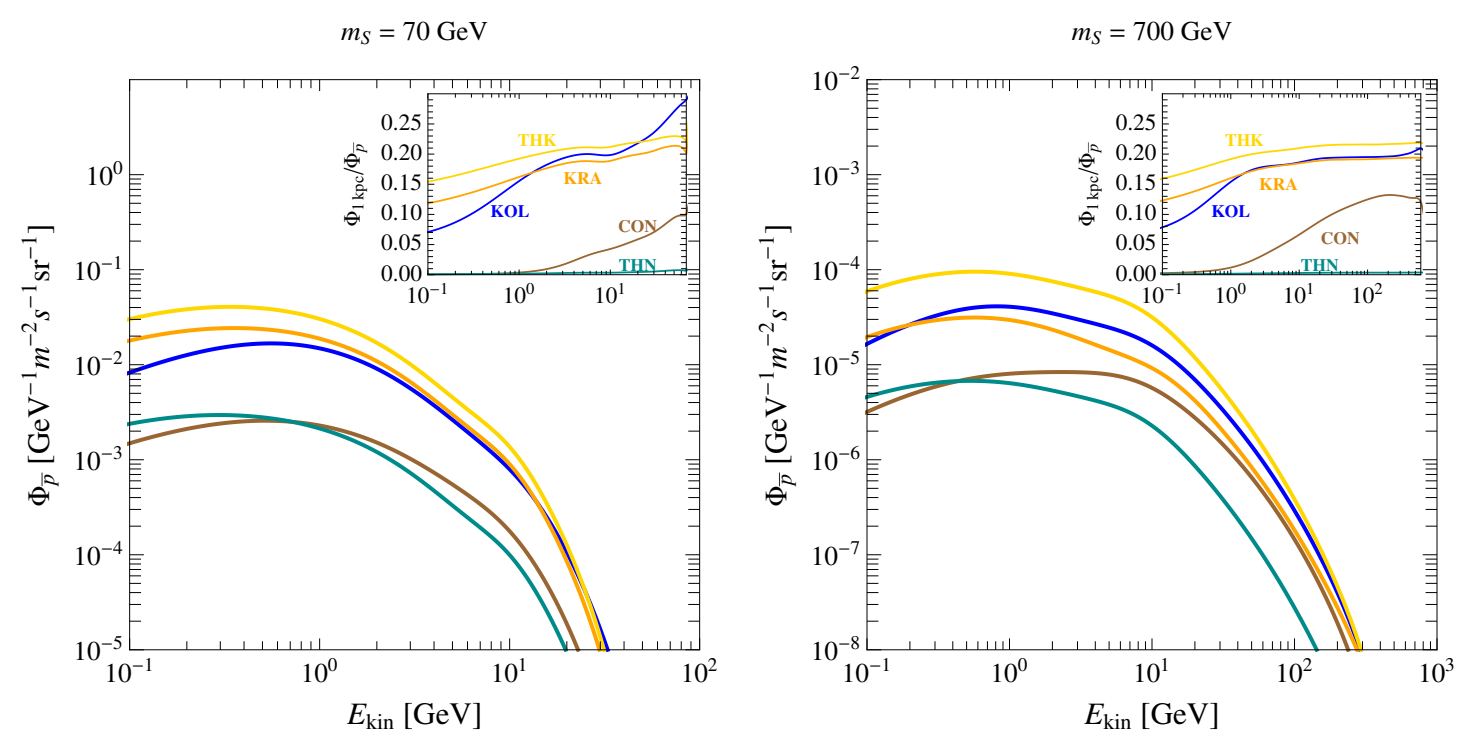

Figure 9. Local antiproton flux from DM annihilation for two representative values of DM mass and the five propagation models defined in table 1 . In the inset plots, we show the relative contribution to the total local flux coming from a region enclosed within $1 \mathrm{kpc}$ from the Galactic center. We use the NFW profile, and we do not include solar modulation effects, $\Phi=0 \mathrm{GV}$.

\section{$5 \quad$ Future perspectives}

In this section we discuss some future perspectives related to our analysis. In section 5.1, we analyze the constraining power of the antiproton data that will be released by the AMS02 experiment. In section 5.2, we analyze the antideuteron flux from DM annihilation in the scalar Higgs portal model.

\subsection{AMS-02}

The Alpha Magnetic Spectrometer (AMS-02) is a particle physics detector hosted on board of the International Space Shuttle, and designed to measure various cosmic-ray fluxes; thanks to this instrument, in the next future a more precise determination of the antiproton and antiproton-to-proton ratio will improve the constraints derived in this paper. To get a more concrete idea, we can simulate the prospects of this experiment by means of a set of mock data, in the energy range of $(1 \mathrm{GeV}, 450 \mathrm{GeV})$. Following ref. [65], we use a linear approximation of the AMS-02 detector energy resolution

$$
\Delta E / E=\left(0.042 \frac{E}{\mathrm{GeV}}+10\right) \%
$$

$[11,18]$. which determines the energy bin-size of the data. Having the bin size $\Delta E_{i}$ and the flux $\mathcal{J}_{i}$ for each bin, the observed antiproton number can be derived as $N_{\bar{p}}=\epsilon a_{\bar{p}} \Phi_{i} \Delta E_{i} \Delta t$, where we take $\epsilon \simeq 1$ for the efficiency, $a_{\bar{p}}=0.2 \mathrm{~m}^{2} \mathrm{sr}$ for the geometrical acceptance of the instrument, and $\Delta t=1$ year for the reference data taking time. Since the dominant statistical uncertainty comes from the antiproton rather than the proton flux, the statistical error is approximately $1 / \sqrt{N_{\bar{p}}}$; for definiteness, we fixed the systematic uncertainties to be 

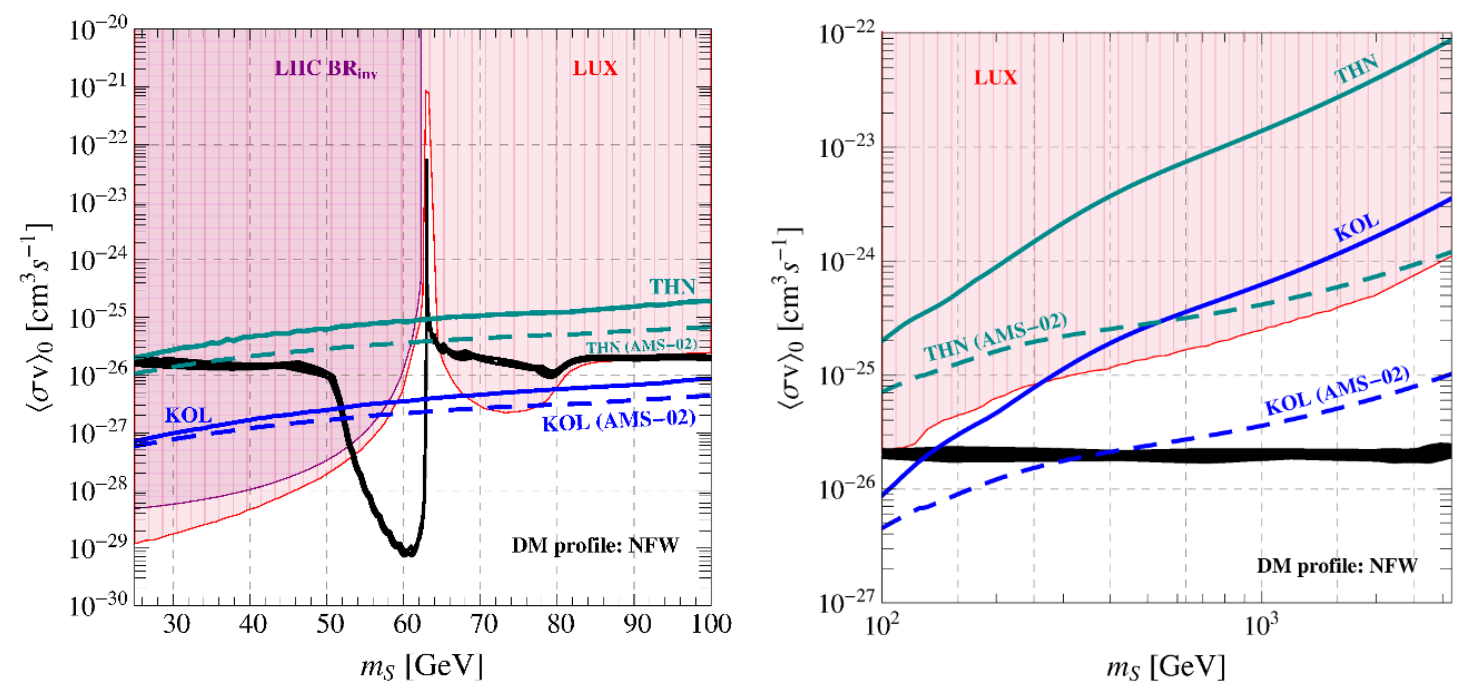

Figure 10. Projected bounds on the annihilation cross-section at zero temperature in the low(left panel) and high-mass region (right panel) considering the THN (dark cyan) and KOL (blue) propagation models. Solid lines represent the current bounds obtained in section 4 while dashed lines are the projected bounds obtained considering mock data for the AMS-02 experiment. The black line matches the observed value of DM relic abundance.

$5 \%$ for one-year data taking. The uncertainty at each data point is the sum in quadrature of the systematic and statistical errors. The central value of the data for each bin, $\mathcal{J}_{i}$, follows the predicted flux from our five benchmark propagation models, which does not contain any DM contribution. With these mock data in hand, we can study the future sensitivity of antiproton-to-proton ratio data on DM models.

In figure 10 we show the projected bounds on the DM annihilation cross-section in the low- (left panel) and high-mass region (right panel) considering, as two extreme cases, the THN and KOL propagation models. We find that the future AMS-02 antiproton data will improve the bound by more than an order of magnitude in the high-mass region. On the contrary, in the low-mass region, our analysis show a little improvement if compared with the existing data. This is because we already exploited the full available data set, which is of reasonably good quality in the low-energy region, and goes until the lowest value of $0.1 \mathrm{GeV}$, while the mock AMS-02 data starts from $1 \mathrm{GeV}$. In the high-energy region, on the contrary, AMS-02's resolution and luminosity are much better than the current status.

\subsection{Antideuteron}

Antideuteron has been proposed in ref. [66] as a promising indirect signal of DM annihilation in the Galactic halo. On a general ground, the annihilation of DM particles into SM hadronic channels - i.e. $q \bar{q}, W^{+} W^{-}$, and $Z Z$ - may produce antideuterons in the final state as a consequence of a two-step process. First - after showering, hadronization, and decay of unstable particles - a large number of antiprotons and antineutrons are produced. Second, an antiproton-antineutron pair may coalesce to form an antideuteron nucleus. The description of this process is usually addressed in the context of the so-called 
coalescence model. Given an antiproton and an antineutron with four-momenta $k_{\bar{p}}^{\mu}$ and $k_{\bar{n}}^{\mu}$, the coalesce model approximates the probability for the formation of an antideuteron with the step function $\Theta\left(\Delta^{2}+p_{0}^{2}\right)$, where $\Delta^{\mu} \equiv k_{\bar{p}}^{\mu}-k_{\bar{n}}^{\mu}$, and $p_{0}$ is the maximum value of relative momentum that allows to form an antideuteron bound state. In this picture, $p_{0}$ is a free parameter and its numerical value has to be extracted from experimental data (see refs. [67, 68] for a detailed discussion). In our analysis we use the results of ref. [69] in order to reconstruct the antideuteron energy spectra produced by DM annihilation. These energy spectra have been obtained using $p_{0}=160 \mathrm{MeV}$, and the coalescence model previously discussed has been applied studying DM annihilations event-by-event [70]. Let us now move to discuss the antideuteron produced by high-energy astrophysical phenomena. The most relevant argument supporting the claim in ref. [66], in fact, emerges from the comparison between the antideuteron signal produced by DM annihilation and the corresponding astrophysical background. To be more precise, there are two key points to keep in mind. i) Antideuterons are produced by high-energy collisions between extragalactic cosmic rays (mostly $p, \bar{p}$ and $\mathrm{He}$ ) and the interstellar gas (mostly $\mathrm{H}$ and $\mathrm{He}$ ) in the Galactic disk; the corresponding cross-section is small and - most importantly — it is characterized by a relatively high kinematical threshold; for instance the energy threshold for the creation of an antideuteron from a collision of a cosmic ray proton (antiproton) with the interstellar gas is $E_{\mathrm{th}}=17 \mathrm{~m}_{p}\left(E_{\mathrm{th}}=7 \mathrm{~m}_{p}\right)$. Let us give a closer look to this numbers considering for definiteness the scattering between a cosmic ray proton and a proton at rest in the interstellar gas. Because of conservation of baryon number, the production of an antideuteron from a proton-proton collision requires a six-body final state, with a total energy square $\hat{s}>\left(6 m_{p}\right)^{2}$. On the other hand, in the rest frame of the gas, $\hat{s}=\left(m_{p}+E_{p}\right)^{2}-k_{p}^{2}$, where $E_{p}$ and $k_{p}$ are the energy and momentum of the cosmic ray proton. From these considerations, it follows that the impinging cosmic ray proton needs to have an energy $E_{p}>17 \mathrm{~m}_{p}$ in order to create an antideuteron. ii) The binding energy for an antideuteron is extremely low, namely $B_{\bar{d}} \approx 2.2 \mathrm{MeV}$. This implies that antideuterons are easily destroyed, and - as a consequence - they do not have to possibility to propagate long enough in order to lose most of their energy. The astrophysical background of antideuterons with kinetic energy $E_{\bar{d}} \lesssim 1-3 \mathrm{GeV}$, therefore, is expected to be extremely small. Below these kinetic energies, an antideuteron flux originated from DM annihilation may easily stand out from the astrophysical background by more than one order of magnitude. In order to translate these qualitative statements into more quantitative results, we need to compare antideuteron background and DM signal after propagation in the Galaxy. The cross-sections describing production, elastic and inelastic scattering of antideuteron - key ingredients in order to solve the corresponding propagation equation, see eq. (3.1) - are not well known. Following ref. [71], we implemented in the DRAGON code all these cross-sections using the results of ref. [72], where they were extrapolated from experimental data under reasonable assumptions. In this way, we are in the position to solve numerically the propagation equation for antideuterons considering both the astrophysical background and the DM signal.

Present and future experiments - two energy bands of the AMS-02 experiment and the three phases of the General AntiParticle Spectrometer (GAPS) [73, 74] — will increase 

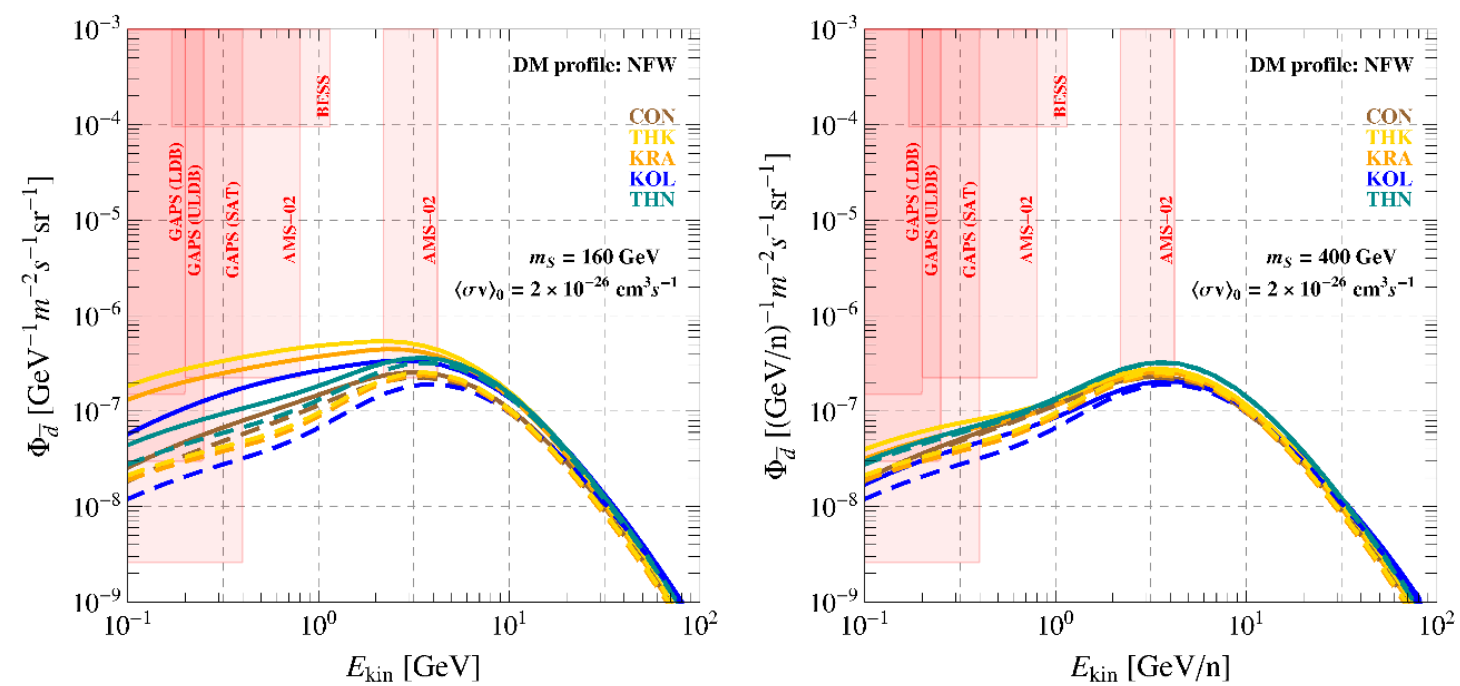

Figure 11. Antideuteron fluxes for $m_{\mathrm{S}}=160 \mathrm{GeV}$ (left panel) and $m_{\mathrm{S}}=400 \mathrm{GeV}$ (right panel), with $\left\langle\sigma v_{\text {rel }}\right\rangle=2 \times 10^{-26} \mathrm{~cm}^{3} \mathrm{~s}^{-1}$. Dashed lines correspond to the background while solid lines to the background plus DM signal. We show the results obtained using the five propagation models in table 1 . The shaded regions give the exclusion by BESS experiment and projected sensitivity of GAPS and AMS-02 experiments (see, e.g., ref. [71]). As a reference value, we fixed the solar modulation potential to be $\Phi=0.25 \mathrm{GV}$.

the sensitivity of searches for cosmic-ray antideuteron over the current bound set by the BESS experiment. For the proposed sensitivities of AMS-02 and GAPS experiments we use the values from ref. [71]. In figure 11, we present the predictions of the scalar Higgs portal model for the antideuteron flux, comparing background and background plus DM signal hypothesis. We analyze two benchmark values for the DM mass, and we use for definiteness the NFW density profile. In the left panel of figure 1 we take $m_{\mathrm{S}}=160 \mathrm{GeV}$, with $\left\langle\sigma v_{\text {rel }}\right\rangle_{0}=2 \times 10^{-26} \mathrm{~cm}^{3} \mathrm{~s}^{-1}$. These values correspond to a DM candidate that, according to figures 4,8 , lies close to the present bound placed by the analysis of the PAMELA antiproton data. We find that the corresponding total antideuteron flux is higher than all the experimental sensitivities of the GAPS experiment assuming the KOL, THK and KRA propagation models. Therefore in these cases, if such DM candidate is realized in Nature, we expect - in principle - a combined detection in both antideuteron and antiproton channels. For illustrative purposes, we show in the right panel of figure 11 a different situation with $m_{\mathrm{S}}=400 \mathrm{GeV},\left\langle\sigma v_{\text {rel }}\right\rangle_{0}=2 \times 10^{-26} \mathrm{~cm}^{3} \mathrm{~s}^{-1}$. These values correspond to a DM candidate that, according to figure 10, lies close to the future bound that will be placed by the analysis of the AMS-02 antiproton data. In this case, however, the total antideuteron flux will be hardly distinguishable from the astrophysical background.

\section{Conclusions and outlook}

In this work, we extracted a new bound on the scalar Higgs portal DM model using highenergy cosmic-ray astrophysics. In summary, the main points of our analysis are the following. First, we studied the propagation equation that governs the motion of charged particles in the Milky Way galaxy using the numerical code DRAGON. This equation 
depends on several free parameters that encode the astrophysical uncertainties describing the propagation of cosmic rays in the Galaxy. We fixed some of these parameters i.e. the halo thickness, the source spectral index, the rigidity slope and the gradient of the convection velocity in the vertical direction - so to define five different propagation setups. Using the measurement of the boron-to-carbon ratio performed by the HEAO-3, ACE, CREAM, ATIC and CRN experiments, we fixed, via a minimization procedure, the remaining ones - i.e. the normalization of the diffusion coefficient, the Alfvén velocity and the low-energy diffusion index. Second, using the propagation setups fixed by the $\mathrm{B} / \mathrm{C}$ data, we predicted the background contribution to the antiproton-to-proton ratio. Finally, we computed the antiproton flux from DM annihilation in the Higgs portal model including three-body final states and QCD radiative corrections; using the antiproton-toproton ratio previously discussed, and combining background and DM signal, we extracted $3-\sigma$ bound on the parameter space of the model. The use of the antiproton-to-proton ratio allowed us to combine different experiments, namely PAMELA, BESS and CAPRICE data. In the antiproton-to-proton ratio, in fact, several systematic effects that plague the comparison between different experiments - as for instance different energy calibration - are integrated out. We compared our antiproton bound with the constraints coming from the LUX and LHC experiments considering - respectively — direct detection of DM particles and the invisible decay width of the Higgs boson. At the same time, we required to reproduce the observed amount of relic density.

We found that the antiproton bound is competitive; in particular, it provides the most stringent constraint on the model in the mass range $m_{\mathrm{S}} \approx 80-300 \mathrm{GeV}$ for most of the analyzed propagation setups. Most importantly, the antiproton bound is the only one able to put in significant tension the resonant region $m_{\mathrm{S}} \approx m_{h} / 2$, otherwise of difficult access to direct detection and collider searches.

In our analysis, we investigated the impact of astrophysical uncertainties related to different propagation setups and different models for the DM density distribution in the Galaxy. Moreover, we discussed future perspectives using a set of mock data in order to simulate those that will be released in the near future by the AMS-02 experiment. Finally, we highlighted in the context of the scalar Higgs portal model the role of the antideuteron channel as an important indirect detection observable able to provide a signature of annihilating DM.

\section{Acknowledgments}

We thank Daniele Gaggero, Andrzej Hryczuk and in particular Piero Ullio for useful discussions and advices. The work of A.U. is supported by the ERC Advanced Grant $\mathrm{n}^{\circ}$ 267985, "Electroweak Symmetry Breaking, Flavour and Dark Matter: One Solution for Three Mysteries" (DaMeSyFla).

\section{A Spin-1/2 Higgs portal}

In this appendix we focus on the following fermionic Higgs portal Lagrangian [75]

$$
\mathcal{L}_{\mathrm{fHP}}=\mathcal{L}_{\mathrm{SM}}+\bar{\chi}\left(i \not \partial-m_{\chi}\right) \chi+\frac{d_{\chi}}{\Lambda}|H|^{2} \bar{\chi} \chi+\frac{i c_{\chi}}{\Lambda}|H|^{2} \bar{\chi} \gamma^{5} \chi
$$



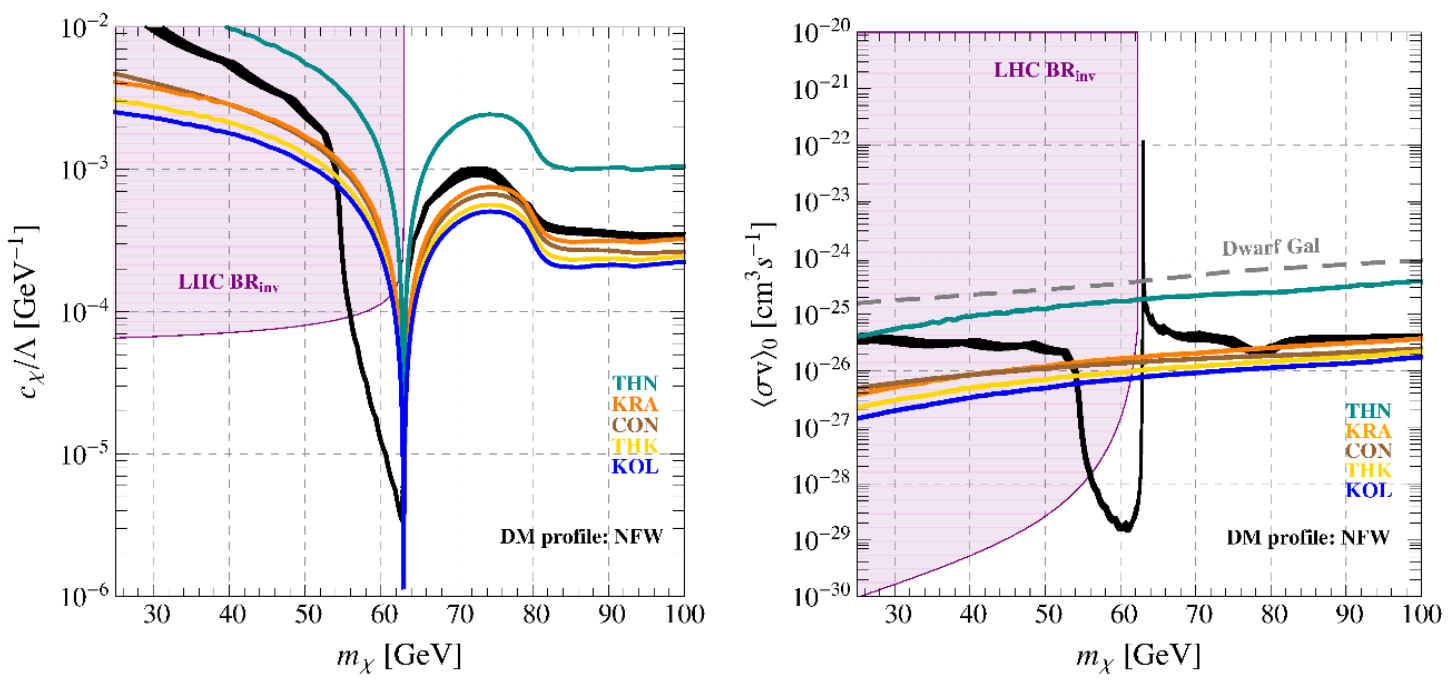

Figure 12. Same as in figure 3 but for the fermionic Higgs portal described in section A.

where $\chi$ is Dirac field playing the role of DM. The parity-conserving interaction $d_{\chi}$ is severely constrained by direct detection experiments [76]; moreover the annihilation crosssection suffers from a p-wave suppression (see, e.g., ref. [77]) that makes it undetectable for any indirect detection experiment. The bound discussed in this paper, therefore, does not apply on this interaction. The parity-violating interaction $c_{\chi}$, on the contrary, induces a velocity-suppressed spin-independent elastic cross-section on nuclei but an unsuppressed annihilation cross-section. As a consequence we expect that the bound from antiprotons, in absence of a significant direct detection signal, is the strongest constraint that can be placed on the parameter space of this model.

Compared with eq. (2.2), the annihilation cross-section times relative velocity is

$$
\sigma v_{\mathrm{rel}}=\frac{1}{\sqrt{s}}\left[\frac{s \lambda_{\chi}^{2} v^{2}}{\left(s-m_{h}^{2}\right)^{2}+\Gamma_{\mathrm{h}, \chi}^{2} m_{h}^{2}}\right] \Gamma_{\mathrm{h}}(\sqrt{s}),
$$

with $\lambda_{\chi} \equiv c_{\chi} / \Lambda, \Gamma_{\mathrm{h}, \chi} \equiv \Gamma_{\mathrm{h}}^{\mathrm{SM}}+\Gamma_{\mathrm{h} \rightarrow \bar{\chi} \chi}$ and

$$
\Gamma_{\mathrm{h} \rightarrow \bar{\chi} \chi}\left(m_{\chi}, \lambda_{\chi}\right)=\frac{m_{h} v^{2} \lambda_{\chi}^{2}}{8 \pi} \sqrt{1-\frac{4 m_{\mathrm{S}}^{2}}{m_{h}^{2}}} .
$$

The phenomenological analysis proceeds parallel to the scalar case.

Open Access. This article is distributed under the terms of the Creative Commons Attribution License (CC-BY 4.0), which permits any use, distribution and reproduction in any medium, provided the original author(s) and source are credited.

\section{References}

[1] O. Chamberlain, E. Segre, C. Wiegand and T. Ypsilantis, Observation of anti-protons, Phys. Rev. 100 (1955) 947 [INSPIRE]. 
[2] M. Kachelriess, P.D. Serpico and M.A. Solberg, On the role of electroweak bremsstrahlung for indirect dark matter signatures, Phys. Rev. D 80 (2009) 123533 [arXiv:0911.0001] [INSPIRE].

[3] P. Ciafaloni and A. Urbano, TeV scale dark matter and electroweak radiative corrections, Phys. Rev. D 82 (2010) 043512 [arXiv:1001.3950] [inSPIRE].

[4] P. Ciafaloni et al., Weak corrections are relevant for dark matter indirect detection, JCAP 03 (2011) 019 [arXiv: 1009.0224] [INSPIRE].

[5] L. Bergstrom, J. Edsjo and P. Ullio, Cosmic anti-protons as a probe for supersymmetric dark matter?, Astrophys. J. 526 (1999) 215 [astro-ph/9902012] [INSPIRE].

[6] A. Barrau et al., Kaluza-Klein dark matter and galactic antiprotons, Phys. Rev. D 72 (2005) 063507 [astro-ph/0506389] [INSPIRE].

[7] P. Chardonnet, G. Mignola, P. Salati and R. Taillet, Galactic diffusion and the anti-proton signal of supersymmetric dark matter, Phys. Lett. B 384 (1996) 161 [astro-ph/9606174] [INSPIRE].

[8] L. Bergstrom, J. Edsjo, M. Gustafsson and P. Salati, Is the dark matter interpretation of the egret gamma excess compatible with antiproton measurements?, JCAP 05 (2006) 006 [astro-ph/0602632] [INSPIRE].

[9] N. Fornengo, L. Maccione and A. Vittino, Constraints on particle dark matter from cosmic-ray antiprotons, JCAP 04 (2014) 003 [arXiv:1312.3579] [INSPIRE].

[10] C. Evoli, I. Cholis, D. Grasso, L. Maccione and P. Ullio, Antiprotons from dark matter annihilation in the Galaxy: astrophysical uncertainties, Phys. Rev. D 85 (2012) 123511 [arXiv: 1108.0664] [INSPIRE].

[11] M. Cirelli and G. Giesen, Antiprotons from dark matter: current constraints and future sensitivities, JCAP 04 (2013) 015 [arXiv:1301.7079] [INSPIRE].

[12] M. Asano, T. Bringmann, G. Sigl and M. Vollmann, 130 GeV gamma-ray line and generic dark matter model building constraints from continuum gamma rays, radio and antiproton data, Phys. Rev. D 87 (2013) 103509 [arXiv:1211.6739] [InSPIRE].

[13] K. Cheung, P.-Y. Tseng and T.-C. Yuan, Cosmic antiproton constraints on effective interactions of the dark matter, JCAP 01 (2011) 004 [arXiv: 1011.2310] [INSPIRE].

[14] K. Cheung, J. Song and P.-Y. Tseng, Cosmic positron and antiproton constraints on the gauge-Higgs dark matter, JCAP 09 (2010) 023 [arXiv:1007.0282] [INSPIRE].

[15] M. Garny, A. Ibarra and S. Vogl, Dark matter annihilations into two light fermions and one gauge boson: General analysis and antiproton constraints, JCAP 04 (2012) 033 [arXiv:1112.5155] [INSPIRE].

[16] D.G. Cerdeno, T. Delahaye and J. Lavalle, Cosmic-ray antiproton constraints on light singlino-like dark matter candidates, Nucl. Phys. B 854 (2012) 738 [arXiv:1108.1128] [INSPIRE].

[17] J. Lavalle, Cosmic-ray antiproton constraints on light dark matter candidates, J. Phys. Conf. Ser. 375 (2012) 012032 [arXiv: 1112.0678] [INSPIRE].

[18] A. De Simone, A. Riotto and W. Xue, Interpretation of AMS-02 results: correlations among dark matter signals, JCAP 05 (2013) 003 [arXiv: 1304.1336] [INSPIRE]. 
[19] M. Cirelli, D. Gaggero, G. Giesen, M. Taoso and A. Urbano, Antiproton constraints on the GeV gamma-ray excess: a comprehensive analysis, JCAP 12 (2014) 045 [arXiv:1407.2173] [INSPIRE].

[20] R. Kappl and M.W. Winkler, The cosmic ray antiproton background for AMS-02, JCAP 09 (2014) 051 [arXiv: 1408.0299] [INSPIRE].

[21] T. Bringmann, M. Vollmann and C. Weniger, Updated cosmic-ray and radio constraints on light dark matter: implications for the GeV gamma-ray excess at the galactic center, Phys. Rev. D 90 (2014) 123001 [arXiv: 1406.6027] [INSPIRE].

[22] D. Marzocca and A. Urbano, Composite dark matter and LHC interplay, JHEP 07 (2014) 107 [arXiv: 1404.7419] [INSPIRE].

[23] V. Silveira and A. Zee, Scalar phantoms, Phys. Lett. B 161 (1985) 136 [INSPIRE].

[24] J. McDonald, Gauge singlet scalars as cold dark matter, Phys. Rev. D 50 (1994) 3637 [hep-ph/0702143] [INSPIRE].

[25] C.P. Burgess, M. Pospelov and T. ter Veldhuis, The minimal model of nonbaryonic dark matter: a singlet scalar, Nucl. Phys. B 619 (2001) 709 [hep-ph/0011335] [INSPIRE].

[26] B. Patt and F. Wilczek, Higgs-field portal into hidden sectors, hep-ph/0605188 [INSPIRE].

[27] F.S. Queiroz and K. Sinha, The poker face of the majoron dark matter model: LUX to keV line, Phys. Lett. B 735 (2014) 69 [arXiv: 1404.1400] [INSPIRE].

[28] A. Djouadi, J. Kalinowski and M. Spira, HDECAY: a program for Higgs boson decays in the standard model and its supersymmetric extension, Comput. Phys. Commun. 108 (1998) 56 [hep-ph/9704448] [INSPIRE].

[29] J.M. Cline and K. Kainulainen, Electroweak baryogenesis and dark matter from a singlet Higgs, JCAP 01 (2013) 012 [arXiv:1210.4196] [INSPIRE].

[30] W.-L. Guo and Y.-L. Wu, The real singlet scalar dark matter model, JHEP 10 (2010) 083 [arXiv: 1006.2518] [INSPIRE].

[31] P. Gondolo et al., DarkSUSY: Computing supersymmetric dark matter properties numerically, JCAP 07 (2004) 008 [astro-ph/0406204] [INSPIRE].

[32] P. Gondolo et al., DarkSUSY webpage, http://www.darksusy.org.

[33] Planck collaboration, P.A.R. Ade et al., Planck 2013 results. XVI. Cosmological parameters, Astron. Astrophys. 571 (2014) A16 [arXiv:1303.5076] [INSPIRE].

[34] ATLAS collaboration, Search for invisible decays of a Higgs boson produced in association with a $Z$ boson in ATLAS, ATLAS-CONF-2013-011 (2013).

[35] CMS collaboration, Search for the Higgs boson decaying to invisible particles produced in association with $Z$ bosons decaying to bottom quarks, CMS-PAS-HIG-13-028 (2013).

[36] A. Falkowski, F. Riva and A. Urbano, Higgs at last, JHEP 11 (2013) 111 [arXiv:1303.1812] [INSPIRE].

[37] LUX collaboration, D.S. Akerib et al., First results from the LUX dark matter experiment at the Sanford Underground Research Facility, Phys. Rev. Lett. 112 (2014) 091303 [arXiv: 1310.8214] [INSPIRE].

[38] K.M. Ferriere, The interstellar environment of our galaxy, Rev. Mod. Phys. 73 (2001) 1031 [astro-ph/0106359] [INSPIRE]. 
[39] C. Evoli, D. Gaggero, D. Grasso and L. Maccione, Cosmic-ray nuclei, antiprotons and gamma-rays in the galaxy: a new diffusion model, JCAP 10 (2008) 018 [arXiv:0807.4730] [INSPIRE].

[40] D. Gaggero, L. Maccione, G. Di Bernardo, C. Evoli and D. Grasso, Three-dimensional model of cosmic-ray lepton propagation reproduces data from the alpha magnetic spectrometer on the International Space Station, Phys. Rev. Lett. 111 (2013) 021102 [arXiv:1304.6718] [INSPIRE].

[41] J.J. Engelmann, P. Ferrando, A. Soutoul, P. Goret and E. Juliusson, Charge composition and energy spectra of cosmic-ray for elements from Be to NI - Results from HEAO-3-C2, Astron. Astrophys. 233 (1990) 96 [INSPIRE].

[42] G.A de Nolfo et al., Observations of the $L i, B e$, and B isotopes and constraints on cosmic-ray propagation, Adv. Space Res. 38 (2006) 1558 [astro-ph/0611301] [INSPIRE].

[43] N.E. Yanasak et al., Measurement of the secondary radionuclides ${ }^{10} \mathrm{Be},{ }^{26} \mathrm{Al},{ }^{36} \mathrm{Cl},{ }^{54} \mathrm{Mn}$, and ${ }^{14} \mathrm{C}$ and implications for the galactic cosmic-ray age, Astrophys. J. 563 (2001) 768.

[44] H.S. Ahn et al., Measurements of cosmic-ray secondary nuclei at high energies with the first flight of the CREAM balloon-borne experiment, Astropart. Phys. 30 (2008) 133 [arXiv:0808.1718] [INSPIRE].

[45] A.D. Panov et al., Relative abundances of cosmic ray nuclei $B-C-N-O$ in the energy region from $10 \mathrm{GeV} / n$ to $300 \mathrm{GeV} / n$. Results from ATIC-2 (the science flight of ATIC), arXiv:0707.4415 [INSPIRE].

[46] D. Mueller et al., Energy spectra and composition of primary cosmic rays, Astrophys. J. 374 (1991) 356 .

[47] BESS collaboration, S. Orito et al., Precision measurement of cosmic ray anti-proton spectrum, Phys. Rev. Lett. 84 (2000) 1078 [astro-ph/9906426] [INSPIRE].

[48] Y. Asaoka et al., Measurements of cosmic ray low-energy anti-proton and proton spectra in a transient period of the solar field reversal, Phys. Rev. Lett. 88 (2002) 051101 [astro-ph/0109007] [INSPIRE].

[49] WiZARd/CAPRICE collaboration, M. Boezio et al., The cosmic ray anti-proton flux between $3 \mathrm{GeV}$ and $49 \mathrm{GeV}$, Astrophys. J. 561 (2001) 787 [astro-ph/0103513] [INSPIRE].

[50] O. Adriani et al., Measurement of the flux of primary cosmic ray antiprotons with energies of $60 \mathrm{MeV}$ to $350 \mathrm{GeV}$ in the PAMELA experiment, JETP Lett. 96 (2013) 621 [INSPIRE].

[51] P. Ciafaloni et al., On the importance of electroweak corrections for Majorana dark matter indirect detection, JCAP 06 (2011) 018 [arXiv:1104.2996] [INSPIRE].

[52] T. Sjöstrand, S. Mrenna and P.Z. Skands, A brief introduction to PYTHIA 8.1, Comput. Phys. Commun. 178 (2008) 852 [arXiv:0710.3820] [INSPIRE].

[53] PYTHIA 8.1 web-page.

[54] J.F. Navarro, C.S. Frenk and S.D.M. White, The structure of cold dark matter halos, Astrophys. J. 462 (1996) 563 [astro-ph/9508025] [INSPIRE].

[55] G. Di Bernardo, C. Evoli, D. Gaggero, D. Grasso and L. Maccione, Cosmic ray electrons, positrons and the synchrotron emission of the galaxy: consistent analysis and implications, JCAP 03 (2013) 036 [arXiv:1210.4546] [INSPIRE]. 
[56] Fermi-LAT collaboration, M. Ackermann et al., Dark matter constraints from observations of 25 Milky Way satellite galaxies with the Fermi Large Area Telescope, Phys. Rev. D 89 (2014) 042001 [arXiv: 1310.0828] [INSPIRE].

[57] Fermi-LAT collaboration, B. Anderson, A search for dark matter annihilation in dwarf spheroidal galaxies with pass 8 data, talk given at the $5^{\text {th }}$ International Fermi Symposium, October 20-24, Nagoya, Japan (2014).

[58] L. Feng, S. Profumo and L. Ubaldi, Closing in on singlet scalar dark matter: LUX, invisible Higgs decays and gamma-ray lines, JHEP 03 (2015) 045 [arXiv:1412.1105] [INSPIRE].

[59] J.F. Navarro et al., The diversity and similarity of cold dark matter halos, Mon. Not. Roy. Astron. Soc. 402 (2010) 21 [arXiv:0810.1522] [INSPIRE].

[60] A.W. Graham, D. Merritt, B. Moore, J. Diemand and B. Terzic, Empirical models for dark matter halos. I. Nonparametric construction of density profiles and comparison with parametric models, Astron. J. 132 (2006) 2685 [astro-ph/0509417] [INSPIRE].

[61] A. Burkert, The Structure of dark matter halos in dwarf galaxies, IAU Symp. 171 (1996) 175 [Astrophys. J. 447 (1995) L25] [astro-ph/9504041] [INSPIRE].

[62] J.F. Navarro et al., The Inner structure of $\Lambda$ CDM halos 3: universality and asymptotic slopes, Mon. Not. Roy. Astron. Soc. 349 (2004) 1039 [astro-ph/0311231] [INSPIRE].

[63] A.W. Graham, D. Merritt, B. Moore, J. Diemand and B. Terzic, Empirical models for dark matter halos. II. Inner profile slopes, dynamical profiles and $\rho / \sigma^{3}$, Astron. J. 132 (2006) 2701 [astro-ph/0608613] [INSPIRE].

[64] A.A. El-Zant, Y. Hoffman, J. Primack, F. Combes and I. Shlosman, Flat-cored dark matter in cuspy clusters of galaxies, Astrophys. J. 607 (2004) L75 [astro-ph/0309412] [INSPIRE].

[65] S. Ting, The alpha magnetic spectrometer experiment on the international space station, talk given at SpacePart12 - The $4^{\text {th }}$ International Conference on Particle and Fundamental Physics in Space, November 5-7, CERN, Geneva (2012) .

[66] F. Donato, N. Fornengo and P. Salati, Anti-deuterons as a signature of supersymmetric dark matter, Phys. Rev. D 62 (2000) 043003 [hep-ph/9904481] [INSPIRE].

[67] A. Ibarra and S. Wild, Prospects of antideuteron detection from dark matter annihilations or decays at AMS-02 and GAPS, JCAP 02 (2013) 021 [arXiv:1209.5539] [INSPIRE].

[68] N. Fornengo, L. Maccione and A. Vittino, Dark matter searches with cosmic antideuterons: status and perspectives, JCAP 09 (2013) 031 [arXiv:1306.4171] [INSPIRE].

[69] M. Cirelli et al., PPPC \& DM ID: a poor particle physicist cookbook for dark matter indirect detection, JCAP 03 (2011) 051 [Erratum ibid. 1210 (2012) E01] [arXiv:1012.4515] [INSPIRE].

[70] M. Kadastik, M. Raidal and A. Strumia, Enhanced anti-deuteron dark matter signal and the implications of PAMELA, Phys. Lett. B 683 (2010) 248 [arXiv:0908.1578] [INSPIRE].

[71] A. Hryczuk, I. Cholis, R. Iengo, M. Tavakoli and P. Ullio, Indirect detection analysis: wino dark matter case study, JCAP 07 (2014) 031 [arXiv: 1401.6212] [INSPIRE].

[72] R. Duperray et al., Flux of light antimatter nuclei near Earth, induced by cosmic rays in the Galaxy and in the atmosphere, Phys. Rev. D 71 (2005) 083013 [astro-ph/0503544] [INSPIRE]. 
[73] K. Mori et al., A novel antimatter detector based on x-ray deexcitation of exotic atoms, Astrophys. J. 566 (2002) 604 [astro-ph/0109463] [INSPIRE].

[74] H. Fuke et al., Current status and future plans for the General AntiParticle Spectrometer (GAPS), Adv. Space Res. 41 (2008) 2056 [inSPIRE].

[75] L. Lopez-Honorez, T. Schwetz and J. Zupan, Higgs portal, fermionic dark matter and a standard model like Higgs at 125 GeV, Phys. Lett. B 716 (2012) 179 [arXiv:1203.2064] [INSPIRE].

[76] S. Kanemura, S. Matsumoto, T. Nabeshima and N. Okada, Can WIMP dark matter overcome the nightmare scenario?, Phys. Rev. D 82 (2010) 055026 [arXiv:1005.5651] [INSPIRE].

[77] W.-C. Huang, A. Urbano and W. Xue, Fermi bubbles under dark matter scrutiny part II: particle physics analysis, JCAP 04 (2014) 020 [arXiv:1310.7609] [INSPIRE]. 